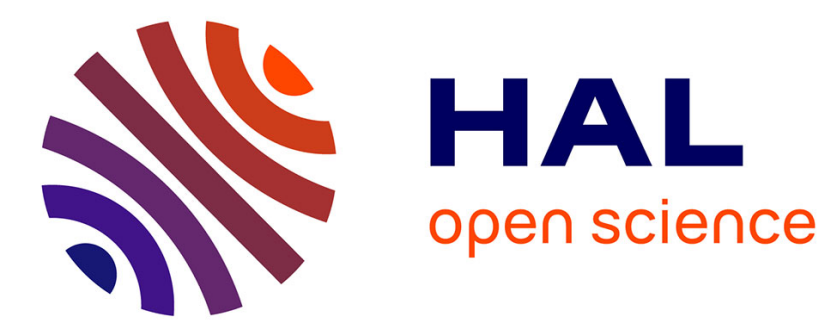

\title{
Ion irradiation of carbonaceous chondrites: A new view of space weathering on primitive asteroids
}

\author{
C. Lantz, R. Brunetto, M. Barucci, S. Fornasier, D. Baklouti, J. Bourçois, M.
} Godard

\section{- To cite this version:}

C. Lantz, R. Brunetto, M. Barucci, S. Fornasier, D. Baklouti, et al.. Ion irradiation of carbonaceous chondrites: A new view of space weathering on primitive asteroids. Icarus, 2017, 285, pp.43 - 57. 10.1016/j.icarus.2016.12.019 . hal-01427337

\section{HAL Id: hal-01427337 https://hal.sorbonne-universite.fr/hal-01427337}

Submitted on 5 Jan 2017

HAL is a multi-disciplinary open access archive for the deposit and dissemination of scientific research documents, whether they are published or not. The documents may come from teaching and research institutions in France or abroad, or from public or private research centers.
L'archive ouverte pluridisciplinaire HAL, est destinée au dépôt et à la diffusion de documents scientifiques de niveau recherche, publiés ou non, émanant des établissements d'enseignement et de recherche français ou étrangers, des laboratoires publics ou privés. 


\title{
Ion irradiation of carbonaceous chondrites: a new view of space weathering on primitive asteroids
}

\author{
C. Lantz $^{1}$, R. Brunetto ${ }^{2}$, M.A. Barucci ${ }^{1}$, S. Fornasier ${ }^{1}$, D. Baklouti ${ }^{2}$, J. Bourçois ${ }^{3}$,
} M. Godard ${ }^{3}$

${ }^{1}$ Laboratoire d'Étude Spatiales et d'Instrumentation en Astrophysique (LESIA)

- Observatoire de Paris, PSL Research University, CNRS (UMR 8109)/ UPMC, Sorbonne Universités / Univ. Paris Diderot, Sorbonne Paris Cité, 92195 Meudon Cedex, France

2 Institut d'Astrophysique Spatiale (IAS), UMR 8617 CNRS/ Univ. Paris Sud, Univ. Paris-Saclay, Bâtiment 104, 91405 Orsay Cedex, France

${ }^{3}$ Centre de Sciences Nucléaire et de Sciences de la Matière (CSNSM), UMR 8609 CNRS/IN2P3 - Univ. Paris Sud, Univ. Paris-Saclay, Bâtiment 104, 91405 Orsay Cedex, France

Submitted to Icarus: April 1st 2016

e-mail: cateline.lantz@obspm.fr, fax: +33145077144; phone: +33145077710 Manuscript pages: 60; Figures: 17; Tables: 3

Running head: Ion irradiation of carbonaceous chondrites

Send correspondence to:

Cateline Lantz

LESIA - Observatoire de Paris

Bâtiment 17

5, place Jules Janssen 92195 Meudon Cedex France

e-mail: cateline.lantz@obspm.fr

phone: +33145077710 


\title{
Ion irradiation of carbonaceous chondrites: a new view of space weathering on primitive asteroids
}

\author{
C. Lantz $^{1}$, R. Brunetto ${ }^{2}$, M.A. Barucci ${ }^{1}$, S. Fornasier ${ }^{1}$, D. Baklouti ${ }^{2}$, J. Bourçois ${ }^{3}$,
}

M. Godard ${ }^{3}$

\begin{abstract}
We present an experimental study on ion irradiation of carbonaceous chondrites, simulating solar wind irradiation on primitive asteroids, to better constrain the space weathering processes of low albedo objects. The irradiations were performed on pressed pellets of the CV Allende, CO Frontier Mountain 95002 and Lancé, CM Mighéi, CI Alais, and ungrouped Tagish Lake meteorites, as well as on some silicate samples (olivine and diopside). We used $40 \mathrm{keV} \mathrm{He}^{+}$with fluences up to $6 \cdot 10^{16}$ ions $/ \mathrm{cm}^{2}$ corresponding to timescales of $10^{3}-10^{4}$ years for an object in the Main Belt. Reflectance spectra were acquired ex situ before and after irradiations in the visible to mid-infrared range $(0.4-16 \mu \mathrm{m})$. Several spectral modifications are observed. In the MIR range, we observe a shift of the phyllosilicates (near 3 and $10 \mu \mathrm{m}$ ) and silicates (near $10 \mu \mathrm{m}$ ) bands toward longer wavelength. In the visible-NIR range, spectral darkening and reddening are observed for some samples, while others show spectral brightening and blueing. Results are also compared with preyious irradiation on ordinary and carbonaceous chondrites. We find that the spectral modifications in the visible range are correlated with the initial albedo/composition. We propose a model for space weathering effects on low albedo objects, showing that those with initial albedo between 5 and $9 \%$ shall not suffer SpWe effects in the visible range. These experiments provide new clues on spectroscopic features modifications within the visible-infrared ranges that could be detected in situ by future sample return missions (Hayabusa-2/JAXA and OSIRIS-REX/NASA).
\end{abstract}

Keywords: Asteroids, Surfaces, Spectroscopy, Meteorites, Experimental techniques 


\section{Introduction}

Surface alteration processes - also known as space weathering ( $\mathrm{SpWe})$, i.e. ion irradiation and micrometeorite impacts - on atmosphere-less small bodies induce variations of the spectral properties of surface materials, thus preventing the identification of sub-surface primitive materials preserved since Solar System formation. Understanding how these modifications act on asteroids and change their spectra will cast light on a better compositional interpretation of remote observations, and can provide valuable support for the forthcoming space missions planned to collect samples of primitive objects and bring them back to Earth (Hayabusa-2/JAXA [Takagi et al., 2011] and OSIRIS-REx/NASA [Lauretta, 2015]). Primitive asteroids (C-complex; [Bus \& Binzel, 2002; DeMeo et al., 2009]) are especially studied because they are thought to contain organic compounds and some water, key elements for life on Earth.

SpWe effects on silicates are pretty well understood with the role of nanophase reduced iron particles $\left(\mathrm{npFe}^{0}\right)$, producing darkening and reddening (increasing slope toward longer wavelength) of their surfaces [Clark et al., 2002a; Brunetto et al., 2015] - and observed in situ on S-type asteroids Eros [Clark et al., 2001] and Itokawa [Ishiguro et al., 2007]. However, there is no general conclusion on what is the spectral trend of C-complex asteroids when exposed to SpWe. Dedicated studies based on spectroscopic observations showed both reddening [Lazzarin et al., 2006; Fornasier et al., 2016; Kaluna et al., 2016] and blueing [Nesvorný et al., 2005; Lantz et al., 2013] effects on spectral slopes.

SpWe effects can be simulated in the laboratory using ion (solar wind simulation) or laser (micrometeorite impacts simulation) irradiations on meteorite samples, 
and most of these experiments on primitive materials were studied on few samples at the same time (see Table 1 for a resume). Hiroi et al. [2013] provide the only available example of multiple irradiation showing a differential trend depending on meteorite classes.

Carbonaceous chondrites (CCs) are composed of chondrules (mostly silicate [Lauretta et al., 2006]), Ca-Al-rich inclusions, Fe-, Ni-metal, and fine-grained matrix. The matrix contains the organic matter [Pizzarello et al., 2006] and shows evidence of metamorphism [Huss et al., 2006] or aqueous alteration [Brearley, 2006]. Proportions of these materials will change with the meteorite type. Thus CCs contain various amounts of different compounds coexisting and it is still not clear how these component characteristics determine the response of different CC classes to SpWe. In a previous study, we have already noticed that VIS-NIR spectral modifications are function of albedo [Lantz et al., 2015]. Here we perform a systematic experimental study on ion irradiation of CCs to better constrain the SpWe of dark objects. Thereby we use the same sample preparation, the same irradiation process and the same spectroscopic measurement method for all the studied samples. This allows us to focus on SpWe effects without being strongly affected by variations of other parameters (powder/pellet, grain size, phase angle, or temperature effects) blurring comparisons between different meteorites compositional classes.

This new set of irradiations is dedicated to study the SpWe effects of a wide variety of CCs: CV Allende, CO Frontier Mountain 95002 and Lancé, CM Mighei, CI Alais, and ungrouped Tagish Lake meteorites. We also analyze natural silicates (olivine and diopside) to compare with S-type asteroids and previous 
experiments on ordinary chondrites (OCs) [Brunetto et al., 2006; Fu et al., 2012]. The diopside was not studied as an analog of pyroxene found within OC (the Ca content being very different) but was an easily available sample to test our experimental set up. We expanded on the work of previous studies [Brunetto et al., 2014; Lantz et al., 2015] by adding an additional irradiation step at a higher dose and also included a new sample of Allende. This sample will allow comparison with our previous results on Allende and validate our new experimental setup.

\section{Experimental techniques}

We crushed the silicate and CC samples into powder using agate mortar and pestle without constraining the grains size (range 1-100 $\mu \mathbf{m})$ and we prepared pellets by pressing $\sim 100 \mathrm{mg}$ of meteorite on a $\mathrm{KBr}$ substrate (samples are held vertically; diameter $13 \mathrm{~mm})$. KBr only supports the meteorite powder ( 200 $\mu \mathrm{m}$ thickness): no ion (implantation depth is a few hundred nanometers [Brunetto et al., 2014]) nor IR light reach the $\mathrm{KBr}$ substrate interface. Ion irradiation of the pellets was performed on the IRMA platform (CSNSM, Orsay) with $\mathrm{He}^{+}$at $40 \mathrm{keV}$ using the following fluences: $5 \cdot 10^{15}, 1 \cdot 10^{16}, 3 \cdot 10^{16}$ and $6 \cdot 10^{16} \mathrm{ions} / \mathrm{cm}^{2}$. The whole pellet surface is irradiated at each step (additive doses: to reach the yalue of $110^{16}$ we used twice the dose of $5 \cdot 10^{15}$ ions $/ \mathrm{cm}^{2}$, and so on) except a corona of $500 \mu \mathrm{m}$ thick on the pellet edge that was hidden by the sample holder. Irradiation was performed in a vacuum chamber $\left(\mathrm{P} \sim 10^{-7} \mathrm{mbar}\right)$, and the samples were placed under vacuum $\left(\mathrm{P} \sim 10^{-2}\right.$ mbar) between irradiation sessions and ex situ MIR reflectance measurements. 
This sample preparation and irradiation protocol was chosen with respect to our previous studies. Here we only focused on the Helium ions because of experimental time limitations. Helium is the second most abundant ion in solar wind. See Brunetto et al. [2014] and Lantz et al. [2015] for details.

Bidirectional reflectance spectroscopy in the visible (VIS, $0.4-1.05 \mu \mathrm{m}$ ) and near-infrared (NIR, $0.9-2.5 \mu \mathrm{m}$ ) ranges was performed using the INGMAR setup (IrradiatioN de Glaces et Météorites Analysées par Réflectance VIS-IR, IAS-CSNSM/Orsay). Spectra were collected through VIS-NIR fibers coupled to a grating spectrometer in the VIS range (Maya2000Pro, Ocean Optics) and a Fourier Transform spectrometer in the NIR range (Tensor37, Bruker) at ambient temperature. We used a fixed collection angle $\mathrm{e}=15^{\circ}$ and illumination angles $\mathrm{i}=15^{\circ}$ (phase angle was $20^{\circ}$ because of $3 \mathrm{D}$-geometry) in the VIS, or $\mathrm{i}=20^{\circ}$ (phase angle was $15^{\circ}$ ) in the NIR. VIS and NIR spectra are acquired on the same pellet location with spectral resolutions of $0.5 \mathrm{~nm}$ and $16 \mathrm{~cm}^{-1}$ respectively. The collecting spot size is 3-4 mm, larger than the pellet heterogeneity, and thus representative of all constituents of the meteorite (matrix and chondrules). The VIS and NIR spectral ranges were combined by overlapping the spectra, merging the two wavelength regions at the common wavelengths $(0.92-0.98 \mu \mathrm{m})$. Reference spectra were acquired using a Spectralon standard (with $99 \%$ Lambertian reflectance; Labsphere) to calibrate the absolute reflectance of our measurements.

The samples were installed on the sample holder and never moved from there between irradiations and measurements. The sample holder is mounted first on the INGMAR chamber to collect spectra before irradiation, then on the IRMA ion beamline, and finally back again on INGMAR to collect spectra after the first 
irradiation. We repeat this scheme until the last irradiation step. The INGMAR configuration allows to have reproducible measurements: spectra are collected at the same position on the pellet, with an error of less than $100 \mu \mathrm{m}$. Thus, the error estimated on the reflectance is about $1 \%$ in relative value for each sample. This is calculated by changing the collecting spot position on the sample and collecting a spectrum each time, to finally evaluate the reflectance level difference. Within the above mentioned uncertainties, there was no change in the slope.

A micro-analysis was performed from Near to Mid Infrared (NIR-MIR, 2 $-16 \mu \mathrm{m}$ ) at the SMIS beamline (Spectroscopy and Microscopy in the Infrared using Synchrotron; Dumas et al. [2006]) of the Synchrotron SOLEIL (France) at ambient temperature using a microscope (Nicolet iN10, Thermo Scientific) with the internal Globar light source (focused beam of $200 \mu \mathrm{m}$, confocal reflection). Reference spectra were collected on a gold sample and the spectral resolution was $4 \mathrm{~cm}^{-1}$. These measurements were performed after the overall protocol presented above to avoid too many manipulations of the samples from the lab to SOLEIL, back and forth, and we thus only have measurements with the highest fluence of irradiation for this spectral range. Spectra are an average of ten or more measurements acquired on different regions of the sample (on each zone, virgin then irradiated), thus we have to deal with possible heterogeneity of the samples at that scale. Measurements were performed every $100 \mu \mathrm{m}$ and the spot size was $200.200 \mu \mathrm{m}^{2}$ covering a grid. These spectra were analyzed individually to estimate the error in the determination of all band peak positions associated to sample heterogeneity estimated to about $0.02 \mu \mathrm{m}$ ( the spectral resolution is about $0.001 \mu \mathrm{m}$ at $2.7 \mu \mathrm{m}$ and about $0.01 \mu \mathrm{m}$ at $10 \mu \mathrm{m})$. 


\section{Sample characteristics}

Meteorites are grouped according to their mineralogy and chemical compositon: matrix- or chondrules-rich, the amount of metal, carbon (carbonates, graphite, organic material), oxygen, etc. Then the petrographic type is used to describe the thermal history, from a largely aqueously altered object (with phyllosilicates; type 1-2) to one that went through some metamorphism (type up to 6; type 3 remaining pristine) [see a review in Weisberg et al., 2006]. Here are some details for the samples used in this study.

Allende is an oxidized CV3 chondrite displaying non equilibrated mineral clusters [Rubin \& Wasson, 1987]. It contains large chondrules (mm size) and large refractory inclusions within an abundant matrix (40 vol\%). The carbon content is low (0.3 wt\%) [Kerridge, 1985]. Frontier Mountain 95002 (FM 95002) is a CO3 having few or no hydrated material [Rubin \& Wasson, 1987; Keller \& Buseck, 1990; Zolensky et al., 1993]. The matrix is 30-40 vol\%, and the carbon content is about 0.6 wt\% [Pearson et al., 2006]. Lancé (CO3.5 type) has the same overall features.

Mighei, a typical CM2, has small chondrules $(\sim 200 \mu \mathrm{m})$ and refractory inclusions, an abundant matrix ( $\sim 70 \mathrm{vol} \%)$ and lots of hydrated minerals $(\sim 75 \mathrm{vol} \%)$ [Howard et al., 2009]. It contains about 2 wt\% of carbon [Kerridge, 1985], mainly in the form of insoluble organic matter, but the soluble part shows a large diversity of organic compounds [Schmitt-Kopplin et al., 2010]. Alais, as a CI1, has the most abundant matrix (99 vol\%, no chondrule) and largely underwent aqueous alteration with 50-60 vol\% of phyllosilicates [Cloutis et al., 2011a]. The carbon 
content is about $5.4 \mathrm{wt} \%$ [Pearson et al., 2006]. Tagish Lake is an ungrouped meteorite having petrological similarity to the CIs and CMs, eventhough Blinova et al. [2014a] showed it is not a simple mixture of CI and CM material. It also underwent aqueous alteration (it is often described as C2 type) but with different degrees, leading to a great lithological heterogeneity for the Tagish Lake meteorite [Izawa et al., 2010; Blinova et al., 2014b]. However, this peculiar CC has a lower density $\left(1.7 \mathrm{~g} / \mathrm{cm}^{3}\right.$ [Gounelle et al., 2001]) and is the most carbon-rich chondrite (5.5 wt\%) [Grady et al., 2002] and the darkest one.

\section{Results}

\subsection{VIS-NIR}

The CC sample spectra are presented in Fig. 1 and 2 while the case of terrestrial silicates is discussed in Appendix A.

The Allende, Lancé, and FM 95002 meteorite spectra before irradiation look similar in shape: strong UV drop-øff, a maximum of reflectance around $0.6 \mu \mathrm{m}$, and a decrease toward longer wavelengths, accompanied with a $1 \mu \mathrm{m}$ band due to silicates [Cloutis et al., 2011b]. The spectra are bluish because of the sample preparation: pellets have bluer spectra than unpacked powder (see Append. B). The absolute reflectance at $0.55 \mu \mathrm{m}$ (at $20^{\circ}$ phase angle; referred as $\mathrm{R}_{0.55}$ in the following text) is 12,15 and $17 \%$ respectively (see Table 2 for the whole values), in agreement with literature data on $\mathrm{CV}$ and $\mathrm{CO}$ chondrites [Cloutis et al., 2012b,a] (though pellet spectra tend to be brighter than the powder ones; see Append. B). Dark meteorites are also known to show a strong opposition effect: we should expect an increase in the reflectance at lower phase angles [Beck et al., 
2012]. After irradiation, $R_{0.55}$ decreases by about $2 \%$ in absolute reflectance for the three pellets and the slope increases (e.g. a linear fit between 0.55 and $2.45 \mu \mathrm{m}$ on Allende gives a slope of $-0.20 \mu \mathrm{m}^{-1}$ before irradiation and goes to $-0.12 \mu \mathrm{m}^{-1}$ after). We notice darkening and reddening effects on these samples.

The three other CCs (Mighei, Alais, and Tagish Lake) are darker before irradiation ( $\mathrm{R}_{0.55}$ of 5,4 and $2 \%$ respectively) and rich in phyllosilicates (see MIR spectra further), even though we do not see any feature associated to the aqueous alteration process in the visible range (see e.g. Cloutis et al. [2014b] for Mighei spectra having the $0.7 \mu \mathrm{m}$ band). Our Alais spectrum is consistent with those published in Cloutis et al. [2011a]; the same for our Tagish Lake sample compared to Hiroi et al. [2004] data. After irradiation, $\mathrm{R}_{0.55}$ increases by about $1 \%$ in absolute reflectance for these samples. The slope gets slightly bluer on Mighei and Alais, but the effect is strong on Tagish Lake (slope on the $0.55-2.45 \mu \mathrm{m}$ range is $-0.01 \mu \mathrm{m}^{-1}$ before and $-0.13 \mu \mathrm{m}^{-1}$ after irradiation).

The dichotomy between the brightest and darkest CCs are summarized in Fig. 3 where we compare the two endmembers (in brightness level) of our set of samples, FM 95002 and Tagish Lake. With increasing irradiation dose, the reflectance and the slope are affected differently for these objetcs. The effects of darkening and brightening are visible with naked eye on the pellets, as shown in Fig. 4.

The red/blue slope variation is a very well known effect of grain size (see the review/of CCs spectroscopic study by Cloutis et al. [2011a]). Because we did not select a size for the study, our samples represent on average an asteroid surface with a wide distribution of grains (even if all meteorites do not grind up the same way). Irradiation cannot change grain size (from microscopic 
point of view) but it may affect roughness (at nanometric scale). So the variations noticed here before and after irradiation are effects of simulated SpWe.

\subsection{NIR-MIR}

Spectra in the $8-16 \mu \mathrm{m}$ range are dominated by silicate bands, and in the 2.4 $-3.3 \mu \mathrm{m}$ range by the hydrated minerals features. The organics near $3.4 \mu \mathrm{m}$ were hardly detected on our samples. We also recall that, in the MIR, the silicate bands are intense and features appear upside down (reststrahlen peaks) thanks to a rapid increase of refractive index leading to strong surface scattering regime [Salisbury \& D'Aria, 1989].

On the three hydrated chondrites (Mighei, Alais, and Tagish Lake), a large absorption band is seen around $3 \mu \mathrm{m}$ (see Fig. 5): a sharp feature at $2.71 \pm 0.01 \mu \mathrm{m}$ associated to $\mathrm{Mg}$-rich phyllosilicates ( $\mathrm{OH}$ stretching) plus a contribution of adsorbed/interlayer molecular water. This is in agreement with spectra published by Beck et al. [2010] on Mighei and Alais, and Hiroi et al. [2001] and Izawa et al. [2008] on Tagish Lake. After irradiation, the band minimum shifts to 2.80, 2.78, and $2.77 \pm 0.01 \mu \mathrm{m}$, respectively, and the sharp feature disappears.

Spectra before irradiation of Allende, Lancé, and FM 95002 (see Fig. 6) all have the same five peaks, with the olivine bands corresponding to a forsterite number (abundance of magnesium silicate, defined as $\mathrm{Mg} /(\mathrm{Mg}+\mathrm{Fe})$, from 0 to 100) of $\mathrm{Fo} \approx 50-55$ and shifting toward $\mathrm{Fo} \approx 30-35$ after irradiation (olivine bands number 2 and 4 following the calibrations by Hamilton [2010]). Similar values were found with previous measurements of an irradiated Allende sample performed by our team [Brunetto et al., 2014]. The bands seen on Allende are in agreement with those measured by Beck et al. [2014]. On Mighei, Alais and Tagish Lake (see 
Fig. 7), the phyllosilicates dominate the MIR range. Alais has an intense peak at $9.83 \pm 0.01 \mu \mathrm{m}$ (as observed by Beck et al. [2010]) shifting to $10.12 \pm 0.01 \mu \mathrm{m}$ and broadening after irradiation. Mighei presents a broad structure with a maximum at $10.20 \pm 0.01 \mu \mathrm{m}$ shifting to $10.81 \pm 0.01 \mu \mathrm{m}$ after irradiation. The structure is made by the superposition of the phyllosilicates contribution at $9.9 \mu \mathrm{m}$, of the olivine peaks at 10.4 and $11.2 \mu \mathrm{m}$, and possibly of the enstatite at $11.5 \mu \mathrm{m}$, similarly to what we measured on the other CM Murchison [Lantz et al., 2015]. Tagish Lake has two distinct peaks: the first one, similar to that observed on Alais, at $9.82 \pm 0.01 \mu \mathrm{m}$ (due to phyllosilicates) shifting to $10.21 \pm 0.01 \mu \mathrm{m}$ after irradiation, and a second one at $11.53 \pm 0.01 \mu \mathrm{m}$ (probably enstatite too) shifting to $11.56 \pm 0.01 \mu \mathrm{m}$ after irradiation. Alais and Tagish Lake also have a peak around $8.65 \pm 0.03 \mu \mathrm{m}$, and Tagish Lake has two peaks above $13 \mu \mathrm{m}$, all shifting the same way.

The case of terrestrial silicates is also discussed in Appendix A.

Raman spectroscopy (see Brunetto et al. [2014] for details on the Raman technique and data reduction) shows the enlargement and displacement of carbon D and G bands for all the samples, similarly to what has been observed in the case of Allende [Brunetto et al., 2014] and Murchison [Lantz et al., 2015]: there is an amorphization of the poly-aromatic structure. In Fig. 8, we show a spectrum before irradiation and a spectrum of the most disordered component after the highest irradiation dose. One can also notice the amorphization of the silicates as seen on the Lancé sample: the olivine peaks at about 850 and $820 \mathrm{~cm}^{-1}$ almost disappear after irradiation on the Raman spectrum. This doublet is the dominant feature of the olivine Raman spectrum that arises from coupled 
symmetric and asymmetric stretching vibrational modes of the constituent $\mathrm{SiO}_{4}$ tetrahedra [Kuebler et al., 2006]. These modes will be efficient under Raman with an olivine crystalline bone, while an amorphous structure will be hardly detected explaining why the peaks disappear after irradiation. The ion irradiation is known to amorphize silicates, as shown by IR spectroscopy analysis [Demyk et al., 2004].

Raman spectroscopy is a technique that is more sensitive to the aromatic carbon content (and to crystalline phase), explaining that one can see better the carbon bands compared to the olivine ones - as opposed to what is seen with IR spectroscopy. The $D$ and $G$ bands are due to the $\mathrm{sp}^{2}$ sites of carbon atoms, and those have a gap falling in the range of the Raman excitation [see e.g. Ferrari \& Robertson, 2001, and references therein]. The Raman cross section being much larger for aromatic carbon than for minerals, the carbon $D$ and $G$ bands dominate the spectra once few aromatic carbons are present [see e.g. Fig.4 in Brunetto et al., 2011].

\section{Discussion}

\subsection{The silicate features}

After irradiation of each sample, we observe a systematic of the silicate/phyllosilicate bands around $10 \mu \mathrm{m}$ toward a spectral region associated with iron-richer minerals (using calibrations on olivine by Hamilton [2010]). The same effect is observed on the aqueous alteration band at $2.7 \mu \mathrm{m}$ (hydrated minerals only seen on Mighei, Alais, and Tagish Lake). These results confirm what we saw on Allende [Brunetto et al., 2014] and Murchison [Lantz et al., 2015], and the new measurement on 
Tagish Lake is in agreement with the study by Vernazza et al. [2013] who also noticed the shift and a certain broadening of the MIR bands for this meteorite. The spectral shift of the IR bands could be due to a loss of magnesium, as a result of preferential sputtering (a differential sputtering of atoms near the surface; the volatile $\mathrm{Mg}$ being more easily sputtered backward than the heavier Fe) [Hapke et al., 1975] possibly leading to amorphization (seen thanks to the broadening of the bands, and confirmed by Raman) [Brucato et al., 2004; Demyk etal., 2004], or preferential amorphization of Mg-rich olivine. See Brunetto et al. [2014] for the full discussion. Because of preferential sputtering, there is more $\mathrm{Mg}$ loss compared to $\mathrm{Fe}$, but $\mathrm{Fe}$ is also a bit sputtered and, as it is heavier, it can be more easily deposited on the surface (e.g. after vaporization/condensation process) under the form of $n \mathrm{pFe}^{0}$. Interestingly, in a new experiment of ion irradiation of Tagish Lake, Dukes et al. [2015] do not see a loss of Mg by using XPS (X-ray photo-electron spectroscopy), while experiments on olivine showed $\mathrm{Mg} / \mathrm{Si}$ ratio decrease [Demyk et al., 2001; Carrez et al., 2002].

The bottom panels of Fig. 6, 7, and 16 show the shift for each band as a function of the initial position. It is interesting to note that the brightest CCs, i.e. Allende, Lancé, and FM 95002, have the same shift for similar bands. We note that the shift on CCs (Fig. 6) is smaller than on the olivine sample (Fig. 16): this is because olivine before irradiation has a higher forsterite number, so that more Mg-minerals can be amorphized/sputtered than the CCs. However, one has to remember that in meteorites the olivine is not pure. On the darkest and hydrated CCs, phyllosilicate structures are efficiently modified by irradiation seen as clear broadening of the bands. One can see that the shift on Mighei is bigger than on Alais or Tagish Lake: this might be due to a different initial compo- 
sition of the phyllosilicates. Indeed, the Mighei spectrum clearly differs from the two others even before irradiation.

With the Fig. 9, we confirm that a relation exists between the measured band shift and the reflectance of each band for a sample: the stronger the absorption, the larger the band shift. We already noticed this trend during our previous study dedicated to Allende [Brunetto et al., 2014]. We proposed that it is due to the balance between ion penetration depth and IR probe depth. A material having a strong imaginary index, like olivine in its strongest IR bands, almost acts like a mirror and a surface scattering regime takes place. So photons in the strongest bands probe a thin layer that is affected by irradiation. The smaller bands have photons emerging from a deeper layer that is not fully modified by irradiation and effects are less noticeable. As an interesting fact, we notice that the most intense peak of olivine (on CCs or terrestrial olivine sample; corresponding to band number 3 ) exceeds the position of a crystalline olivine with a forsterite number of 0 (no $\mathrm{Mg}$ content $=100 \%$ fayalite) [Hamilton, 2010]: this is because $\mathrm{Mg}$ loss is also accompanied by amorphization [Demyk et al., 2001; Carrez et al., 2002].

One has to note that these band shifts do not disagree with the possible formation of nanophase reduced iron particles $\left(\mathrm{npFe}^{0}\right)$ [Hapke, 2001]: the shift toward Fe-rich spectral region could also be due to these $\mathrm{npFe}^{0}$ (enrichment of $\mathrm{Fe}$ ). As a matter of fact, Dukes et al. [2015] saw an increase of the Fe/Si ratio on their Tagish Lake sample after ion irradiation. Keller et al. [2015b] and Matsuoka et al. [2015] did not see any shift of the NIR and MIR bands, but they both used STEM (Scanning Transmission Electron Microscopy) and observed amorphization of phyllosilicates (serpentine group). In the first case 
the authors observed also a change in the $\mathrm{Fe}^{2+} / \mathrm{Fe}^{3+}$ ratio, with $\mathrm{Fe}^{2+}$ becoming more abundant in the irradiated material, while in the second case, the authors observed S- and Fe-rich small amorphous silicate particles (with diameters 20-1000 $\mathrm{nm}$ ) formed and deposited on the surface after irradiation. On one hand, Matsuoka et al. [2015] performed laser irradiation so the modification process (microstructural and chemical effects) is different (for example melting) and it is hard to compare with our experiment. On the other hand, Keller et al. [2015a] used ion irradiation but at lower energy $\left(1 \cdot 10^{18} \mathrm{He}^{+} / \mathrm{cm}^{2}\right.$ at $\left.4 \mathrm{keV}\right)$ : one can assume that the spectral changes we see, and in particular the $2.7 \mu \mathrm{m}$ band shift, take place in the volume probed by the reflectance and thus require higher ion penetration depth to be seen (a SRIM calculation gives a penetration depth for $40 \mathrm{keV}$ He ions 8.5 times bigger than for $4 \mathrm{keV}$ ). The energy of 40 $\mathrm{keV}$ used in our study is about ten times higher than the slow solar wind energy of helium ions, but it is close to the average slow solar wind energy for heavier ion such as argon. Moreover, higher energy helium ions are emitted from the Sun through active regions and solar flare. Thus, because there is a wide distribution of energies and ions in the solar wind, we should detect this shift also on asteroids. For example, Matsumoto et al. [2015] proposed that $14 \mathrm{keV} \mathrm{He}^{+}$might have played a role in the formation of a thick spaceweathered layer they detected on an Itokawa particle.

The amount of available data on asteroids in the NIR-MIR has increased since the last decade [see a review in Reddy et al., 2015], although limited by atmospheric transmission. The $2.7 \mu \mathrm{m}$ band lies within the wavelength range of strong absorption by water vapor in Earth atmosphere [e.g. Takir \& Emery, 2012], 
thus it will be impossible to use this spectroscopic information of SpWe from remote sensing observations. The thermal region near $10 \mu \mathrm{m}$ is even less studied from the ground and data are prone to errors due to highly variable telluric ozone structure $(9.2-10.2 \mu \mathrm{m})$ [Lim et al., 2005] and requires the use of space telescopes like Spitzer did [Emery et al., 2005]. These SpWe effects on MIR spectra will be hardly seen with telescopic observations, but they could be used during the characterization of asteroid surfaces by space missions and help choosing sampling sites. For example, we detect band shifts of at least $50 \mathrm{~nm}$ at $2.7 \mu \mathrm{m}$ : resolution of VIR onboard Dawn/NASA is $10 \mathrm{~nm}$ at this wavelength [de Sanctis et al., 2011] and is about $20 \mathrm{~nm}$ for OVIRS on OSIRIS-REx/NASA [Reuter \& Simon-Miller, 2012] and NIRS3 on Hayabusa-2/JAXA. Around $10 \mu \mathrm{m}$, resolution of OTES on OSIRIS-REx spacecraft is $10 \mathrm{~cm}^{-1}$ [Hamilton \& Christensen, 2014], that is about $10 \mathrm{~nm}$ while we dectect band shift of at least $20 \mathrm{~nm}$ for the most intense bands. In the future, the James Webb Space Telescope could provide more numerous and accurate data for both NIR and MIR ranges.

\subsection{The darkest surfaces case}

\subsubsection{VIS-NIR trends for irradiated CCs as a function of the initial composition}

With the new experiment, we obtain and we confirm the VIS-NIR darkening/reddening trend for the $\mathrm{CO}$ and $\mathrm{CV}$ types of carbonaceous chondrites [Lazzarin et al., 2006; Brunetto et al., 2014]. Our new irradiations of olivine and diopside also confirm this very well-known effect of ion/laser irradiation on silicates and OCs [Yamada et al., 1999; Sasaki et al., 2001, 2002; Brunetto \& Strazzulla, 2005; Marchi et al., 2005; Strazzulla et al., 2005; Brunetto et al., 2006; Loeffler et al., 2009; Fu et al., 2012] (see Append. A). The importance of $n p F e^{0}$ in these 
modifications has been underlined by many authors [Hapke, 2001; Noble et al., 2007] and their presence confirmed on S-type NEA Itokawa (Hayabusa/JAXA mission) [Hiroi et al., 2006; Yoshikawa et al., 2007] as a result of solar wind implantation accompanied with amorphization of target minerals and redeposition of sputtered materials [Noguchi et al., 2011, 2014]. Finally, here we show that Mighei, Alais and Tagish Lake become brighter and bluer after SpWe simulation. The response to SpWe of the darkest CCs is obviously different.

In our previous attempt dedicated to understand $\mathrm{SpWe}$ effects on primitive asteroids, and considering other studies, we proposed a composition dependent trend [Lantz et al., 2015], to explain the non convergent conclusion on reddening/blueing and darkening/brightening debate. If we strictly compare experiments done in the same way, the new set of irradiations made here allows us to separate the effect of the initial composition from other effects such as sample preparations, experimental setups, and viewing geometries. Then, we focus on the weathering effect by performing the ratio of altered spectrum divided by the unaltered spectrum. These ratios allow to quickly visualize how the SpWe change the spectra with increasing irradiation: the reflectance ratio is lower than 1 when surface darkens and ratio slope has a positive value if the spectrum reddens. The resulting reflectance and slope of the ratioed spectra are reported in Fig. 10. They indicate that the spectral reponse for SpWe of CCs, and in extent of C-complex asteroids, are related to the initial composition and the carbon content. With higher irradiation doses, some CCs will become redder and darker like OCs (chondrules richer), while the most primitive (matrix and carbon richer) will become bluer and brighter. 
Both the blueing and the brightening behaviors of the darkest surfaces have already been explored by Moroz et al. [2004a] who performed ion irradiation of complex hydrocarbons. The authors explain that carbonization process - hydrogen loss and carbon structural changes (growth of large aromatic clusters) - is responsible of the blueing and brightening effects. The carbonization leads to an optical gap decrease and the material gets metal-like optical properties (the importance of optical gap modifications in hydrocarbon materials is discussed by Jones [2012]). Thus, ion irradiation causes a gradual growth of refractive index imaginary part and surface scattering dominates at small wavelengths: this could explain the reflectance increase in the VIS range. Because the absorption coefficients of bitumens are much lower in the NIR region, the spectral behavior is controlled mostly by volume scattering, and carbonization should lead to darkening in the NIR range. Brunetto \& Roush [2008] performed ion irradiation of methane and derived the optical constants. The imaginary index on the irradiated sample (around $1 \cdot 10^{-2}$ in the VIS-NIR range) is bigger than on pure methane (around $5 \cdot 10^{-4}$ ). The imaginary index value has been calculated for the Tagish Lake meteorite by Roush [2003] and is around $5 \cdot 10^{-3}$ in this region. Thereby, ion irradiation of CCs could lead to a carbonization process of the organic component inducing growth of the imaginary index, and then explain the blueing and brightening effects described by Moroz et al. [2004a]. On Tagish Lake, Dukes et al. [2015]/showed that there is an important loss of $C$ : this could also explain the brightening of the surface.

More recent studies tend also to emphasize the role of initial composition. 
Gillis-Davis et al. [2015] performed laser irradiation (simulation of micrometeorite impact heating) of Allende and Murchison, as well as graphite to investigate the extent to which spectral changes in carbon, as a function of laser irradiation, might be observed in the meteorites. Spectra of laser irradiated Allende responded like OCs and S-type asteroids: decrease of reflectance and reddening of the slope accompanied with mitigation of the bands in the $0.4-2.4 \mu \mathrm{m}$ range, while a blueing effect was observed on the short wavelengths $(<0.55 \mu \mathrm{m})$. Murchison spectra became darker too, with a progressive loss of the $0.7 \mu \mathrm{m}$ band for increasing dose of irradiation, but without significant spectral slope changes. The graphite sample became brighter after some irradiations and then slightly darkened, but it did not show systematic change in the slope. A TEM x-ray mapping showed a carbon phase being deposited within grain boundaries and vesicles. Although Gillis-Davis et al. [2015] presented laser irradiation while we deal here with ion irradiation (the effects would probably differ, and indeed we do not have the same result for surface brightness on the CM sample), they showed that Murchison and Allende exhibit distinct spectral responses to laser SpWe that may be due to the different graphitic carbons content in matrix.

Kaluna \& Gillis-Davis [2015] also used laser irradiation but focused on phyllosilicate minerals, widely present in some $\mathrm{CC}$ classes, and that could also drive the spectral modifications of such primitive materials. They compared two serpentine end-members, cronstedtite $\left(\mathrm{Fe}^{2+}{ }_{2} \mathrm{Fe}^{3+}\left(\mathrm{Si}_{2} \mathrm{Fe}^{3+} \mathrm{O}_{5}\right)(\mathrm{OH})_{4}\right)$ and lizardite $\left(\mathrm{Mg}_{3} \mathrm{Si}_{2} \mathrm{O}_{5}(\mathrm{OH})_{4}\right)$, with olivine (anhydrous silicate; $\left.\left(\mathbf{M g}^{2+}, \mathbf{F e}^{+2}\right)_{2} \mathbf{S i O}_{4}\right)$. Unsurprisingly, the olivine sample became redder and darker in the whole $0.5-2.5 \mu \mathrm{m}$ range. Lizardite became slightly darker in the VIS part (and so reddened). Cronstedtite became also a bit darker in the VIS, but reflectance decreased a lot in the NIR so a blueing 
of the slope was measured. Because $\mu \mathbf{m}$-size (larger than $\mathbf{5 0} \mathbf{~ n m}$ ) iron particles production can lead to darkening without reddening (as opposed to nm-size) [Noble et al., 2007; Lucey \& Riner, 2011], the authors proposed that such particles are created first in the cronstedtite sample to explain the blueing trend. They also postulate the possibility that the continuous darkening in the NIR is due to the creation of an opaque phase, spectrally neutral. CCs of types 1 and 2 (that underwent aqueous alteration process) are mainly made of phyllosilicates with varying amount of Fe and Mg elements [Tomeoka et al., 1989]. In particular, CMs show the widest amount of cronstedtite (40-50\% of detected hydrated minerals) than Mg-rich serpentine (25-30\%) [Howard et al., 2009]. The blueing effect we measure on Mighei, Alais, and Tagish Lake is similar to this cronstedtite blueing under irradiation. One has to note, again, that here we compare laser and ion irradiations. Anyway, Kaluna \& Gillis-Davis [2015] also proved that two Fe-Mg end-member compositions exhibit a divergent set of spectral trends: this would imply that, whatever the technique used for irradiation, initial sample composition influences the modification pathways Our results support these conclusions.

\subsubsection{Carbon vs. $n p F e^{0}$}

However, the role of $\mathrm{npFe}^{0}$ when formed within a dark matrix is not yet understood. The accumulation of these opaque reduced iron particles on semitransparent minerals (like S-type asteroids and OCs) leads to the darkening (these are absorbing in the VIS) and reddening (reflectance remains high in the NIR) effects [Hapke, 2001]. But CCs already have finely-dispersed opaque particles within the matrix (imparting an overall dark appearance) [Cloutis et al., 2011b] and it is possible that opaque materials mask the optical effects of SpWe prod- 
ucts on low-albedo objects [Pieters et al., 2000]. Furthermore, some authors proposed that because npFe ${ }^{0}$ have higher albedos than $\mathrm{C}$-type asteroids (and CCs), one could expect an increase of reflectance plus a blueing toward NIR [Clark et al., 2002a; Rivkin et al., 2002].

It is proved that the carbonaceous phase is modified after irradiation, both from the point of view of the backbone structure with amorphization (probed with Raman spectroscopy; see Fig. 8, Brunetto et al. [2014], and Lantz et al. [2015]) and from the chemistry because $\mathrm{CH}$ bonds are affected by ion irradiation processes [Godard et al., 2011]. We have also shown with MIR spectroscopy that silicates suffer strong modifications like amophization and Mg loss by back-scattered sputtering. Raman and MIR spectroscopies are sensitive to different compounds so that these experimental techniques allow us to distinguish between several effects. In the VIS-NIR range, the aromatic-rich amorphous carbon phase and the $n p F e^{0}$ can have the same effects on the spectra: both have strong absorption properties (higher imaginary index compared to the matrix) leading to absorbing pattern, especially in the VIS, even if they are present in small amount, finely dispersed (small particles with size lower than the wavelength) within a silicate matrix. Hapke [2001] has developed a spectral model to explain the spectral modifications induced by SpWe. This model is based on the Maxwell-Garnett effective medium theory, to calculate the absorption coefficient of a semi-transparent silicate host medium containing inclusions of small metallic iron spheres (the so called $\mathrm{nFFe}^{0}$ ). In the model, this homogeneous medium is then used as a coating layer on top of unaltered materials. It has been widely used to describe the effects of SpWe on reflectance spectra of silicates [Brunetto et al., 2007] and S-type aster- 
oids with the example of Itokawa [Hiroi et al., 2006; Bonal et al., 2015]. We also tested this model using npFe ${ }^{0}$ (optical constants from Johnson \& Christy [1974]) or inclusions of dehydrogenated carbon (Raman spectrocopy proved it becomes amorphous under ion irradiation; with optical constants from Jager et al. [1998]) as contaminating inclusions within the silicate matrix. The test (see Fig. 11) has shown that the spectral changes are very similar and we cannot conclude on which compound is dominating the SpWe effects within the VIS range. However, we notice that it needs more carbon than iron particles to affect the spectra because the absorbing coefficient is higher for iron. The Maxwell-Garnett theory was also used in a model for TNOs by Grundy [2009]: when little carbonaceous particles smaller than the wavelength are mixed with ice (transparent matrix), that material becomes highly absorbing (the $R$ albedo decreases of more than $50 \%$ with $1 \%$ of these particules). The fact that the carbon content has an important role in the SpWe modifications we are simulating is confirmed by the correlation we observe between reflectance variation after irradiation and the carbon content of the meteorites (as shown in Fig. 12). We do need additionnal measurements with other techniques, from the microscopic point of view, to resolve this issue for the role of carbon vs. $\mathrm{nFe}^{0}$.

\section{Implication for SpWe of asteroids}

We propose a simple model of SpWe expected effects on low albedo objects. To do so, we fit the values of reflectance and slopes from the ratio plots (that allow to easily represent the way the spectra change under irradiation) with a linear function depending on the initial value of reflectance at $20^{\circ}$ phase angle 
before irradiation $\left(\mathrm{R}_{0.55}\right)$ :

$$
\mathrm{Val}=a+b \cdot \ln \left(R_{0.55}\right)
$$

where $\mathrm{Val}$ is the ratio value of reflectance at $0.55 \mu \mathrm{m}$ or spectral slopes on the range $0.55-1.05 \mu \mathrm{m}$, as shown in Fig. 13. We present the result of the study for both lower and higher doses of irradiation (ions with $40 \mathrm{keV}$ energy) in Table 3. The most important parameter is the factor $b$ giving an information on the function inclination: it will increase (in absolute value) with increasing irradiation dose (as shown by the comparison of the two doses used). The Fig. 14 shows the evolution of this factor $b$ as a function of the dose for both level of reflectance and slope variation. These fits allow to describe the behavior of CCs under ion irradiation: the brightest members with $\mathrm{R}_{0.55} \geq 4.5-9 \%$ (derived from the model; explanations in Fig. 13) will have a darker surface and a redder slope following the given functions, while the darkest members will have the opposite spectral response.

This model is valid under specific conditions. We underline the different behaviors that CCs have, depending on their initial composition, for a given irradiation fluence and phase angle. The phase angle is an important parameter on the spectral measurements: when it goes to $0^{\circ}$ there is an opposition effect and the reflectance strongly increases [Beck et al., 2012], while when the phase angle grows a spectral reddening has been noticed on asteroids [Clark et al., 2002b], meteorites [Gradie et al., 1980], and comet 67P/ChuryumovGerasimenko [Fornasier et al., 2015]. Furthermore, grain size effect is not negligible: the finer grain, the redder spectrum slope [Johnson \& Fanale, 1973]. Measurements at different temperatures can also lead to differences in the spectra (band shift for example) [Moroz et al., 2000]. 
Brunetto et al. [2014] noticed that the optical effects of irradiation on Allende are correlated with the nuclear energy loss. This kind of information can be useful to rescale the laboratory dose to the astrophysical distribution of ions. We showed that the VIS slope reaches an asymptotic value starting from $3 \cdot 10^{16} \mathrm{He}^{+} / \mathrm{cm}^{2}$ (same energy of $40 \mathrm{keV}$ used here). With the new step of irradiation at $6 \cdot 10^{16} \mathrm{He}^{+} / \mathrm{cm}^{2}$ we can confirm that an asymptote is reached for both VIS slope and $\mathrm{R}_{0.55}$ (see Fig. 3: parameter $a$, giving the asymptote, is very close to the data point of the last fluence) on the brightest CCs. The highest dose we use corresponds to timescales of about $10^{3}-10^{4}$ years for an object at $3 \mathrm{AU}$, and the implantation depth is a few hundred nanometers [Brunetto et al., 2014]. This is consistent with the SpWe rims formation timescale obtained by direct measurements of the exposure to energetic ions of the Itokawa particles brought to Earth by the Hayabusa mission and consisting of thin layer of vapor/sputtered deposited on a larger amorphous layer with $n \mathrm{pFe}^{0}$ and npFeS [Nagao et al., 2011; Noguchi et al., 2014]. Berger \& Keller [2015] derive surface exposure $\leq 10^{5}$ years of three Itokawa grains from solar

\section{flare particle track densities.}

It is less obvious to derive timescales from the three other CCs, the darkest ones, especially for the slope: the asymptote is not clearly reached. One can wonder if this is because these samples are carbon-richer, and that modifications of the carbonaceous phase have higher timescales with respect to $\mathrm{nFFe}^{0}$ formation. Sample return missions targeting low albedo asteroids will provide important clues to solve this issue.

Nevertheless, SpWe features were found on several sides of Itokawa grains [Matsumoto et al., 2015], showing that grain motion due to impacts [Shestopalov et al., 
2013], seismic shaking [Richardson et al., 2005], or YORP effect [Walsh et al., 2008] for example, can be quite efficient to rejuvenate a surface. Furthermore, some regolith can be formed by thermal fatigue [Delbo et al., 2014]. All of these processes are counterbalancing the fast SpWe rate on surface particles.

One of the astrophysical implications of our experimental results is that there must be a large fraction of asteroids whose albedo lies in the 4.5-9\% range, that should not show VIS-NIR variations upon irradiation. We checked for possible confirmation of this prediction. Clark et al. [1999] published photometric results on Mathilde, the darkest asteroid observed by a spacecraft (mission NEAR/NASA), with a normal reflectance values ranging from 0.041 to 0.053 : the authors assessed that the very little color variation seen in Mathilde data (typical normal reflectance constrasts are on the order of $6 \%$ from the mean) indicates that SpWe processes have not altered the optical properties of the surface materials. Considering our new results on spectroscopy of irradiated CCs, this assumption appears plausible because this reflectance value lies within the range of no or weak effects due to SpWe. Let us recall that the Murchison sample we studied previously [Lantz et al., 2015] did not show clear modifications under irradiation: so that Murchison could be the low endmember of the population for which initial composition/albedo does not allow SpWe to be efficient, like what was observed on Mathilde.

Some recent studies tried to highlight SpWe effects from observational data comparing young and old primitive asteroid families [Kaluna et al., 2016; Fornasier et al., 2016] but it appears hard to distinguish between SpWe and other hypothesis like spectral heterogeneity within a family. We shall build stronger statistics 
from the remote sensing observations for dark asteroids and complete the view we have of the phenomenon. Without taking into account the heterogeneity issue, the model could be used to see how the asteroid albedo distribution should change or have changed under SpWe, considering the initial composition as the mean of the population distribution.

\section{Conclusions}

In this paper we presented reflectance spectra of several meteorites probed by visible and infrared spectroscopy. We studied ion irradiation effects as a simulation of the solar wind bombardment of asteroid surfaces. For the first time, the same experiment is done using the same method on six CCs spanning a wide range of initial albedo and chondrules, carbon and hydrated mineral contents. In the VIS-NIR range, the $\mathrm{CV}$ and $\mathrm{CO}$ classes have a similar spectral answer to SpWe than OCs and S-type asteroids (darkening/reddening) while the darkest and most aqueously altered chondrites (CM/CI/ungrouped Tagish Lake) show a clear surface brightening and a certain blueing. It confirms that a relationship exists between the spectral variations observed and the initial composition [Lantz et al., 2015]. As already noticed in Brunetto et al. [2014] and Lantz et al. [2015], there is a systematic shift of phyllosilicate and olivine bands near 2.7 and $10 \mu \mathrm{m}$ toward Fe-rich spectral region (longer wavelengths) that may be attributed to the amorphization and/or loss of Mg due to preferential sputtering, and/or presence of $n p F e^{0}$.

The formation of these $n \mathrm{pFe}^{0}$ are the common thought mechanism for space weathering on S-type asteroids in the VISNIR range, but there is a significant 
heterogeneity in $\mathrm{CC}$ compositions influencing the reflectance effects measured here. On one hand, we propose that because the $\mathrm{CO} / \mathrm{CV}$ chondrites are mainly composed of chondrules (typically made of Si compounds), their spectral answer is close to that of OCs and S-type asteroids with predominating $n \mathrm{pFe}^{0}$ action. On the other hand, the darkest chondrites are matrix dominated: the carbonaceous phase could dominate the optical effects, and we do observe modifications as a clear linear relationship exists between the albedo variation upon irradiation and the carbon content of the meteorite. Finally, the role of hydrated minerals can not be excluded, and the influence of $\mathrm{npFe}^{0}$ on the darkest minerals reflectance response have not yet been explored.

Our global study on several CCs suggests an empirical model of SpWe effects on the darkest meteorites indicating clearly that the initial composition rules the trends on reflectance and slope in the VIS-NIR. We predict that asteroids initially darker than $5 \%$ become brighter by about $0.5-1.5 \%$ and bluer, while asteroids brighter than $9 \%$ become darker by about 1.5-3\% and redder like OCs and S-types - our model can explain the absence of albedo variations osberved on asteroid Mathilde - at short timescales $\left(10^{3}-10^{4}\right.$ years $)$ consistent with Itokawa observations. This implies that resurfacing processes must be also very efficient, as suggested by recent studies on regolith motions. However, SpWe studies must be pursued to decipher the physical, chemical and mineralogical changes it causes at nanometric scale.

\section{Acknowledgments}

We warmly thank the Natural History Museum of Vienna (Dept. of Mineralogy and Petrography; Lancé sample NHMW_ID_3593_[3] (von A958) and Al- 
lende sample NHMW_ID_413_[5]), the Vatican Observatory (Alais and Mighei samples), J. Brucato (Tagish Lake sample), and L. Folco (FM 95002 sample) for providing us with the meteorite samples, and C. Engrand for the silicates samples. We also thank C. Bachelet and J. Duprat for the access to the IRMA facility, and O. Mivumbi and P. Duret for their help on INGMAR. We are grateful to A. Arondel, P. Blache, S. Blivet, D. Ledu, and F. Fortuna for their contribution to the conception of the experimental setup, to F. Borondics and C. Sandt for their help and support at the SMIS beamline of the Synchrotron SOLEIL, and to P. Beck, L. Bonal, and E. Quirico for useful discussions. We especially thank E. Dartois and F.E. DeMeo for the insightful suggestions improving the text. We thank the two anonymous referees for useful comments.

This research is part of a joint IAS-CSNSM project (INGMAR) and it has been funded by the French national program Programme National de Planétologie (PNP), by the Faculté des Sciences d'Orsay, Université Paris-Sud (Attractivité 2012), by the French National Research Agency Agence Nationale de la Recherche (contract ANR-11-BS56-0026, OGRESSE), and by the P2IO LabEx (ANR-10-LABX-0038) in the framework Investissements d'Avenir (ANR-11-IDEX-0003-01) managed by the French National Research Agency (ANR). C.L., M.A.B., and S.F. acknowledge the funding of the French national space agency Centre National d'Études Spatiales (CNES).

\section{Appendix A. The silicate samples}

Before irradiation, the VIS-NIR spectra (see Fig. A1) of olivine and diopside are fully consistent with what is expected for these silicates in terms of reflectance level (68 and $56 \%$ respectively at $0.55 \mu \mathrm{m})$ and band shape [Clark et al., 2007]. 
The olivine shows a weak band at $0.65 \mu \mathrm{m}$, and the broad and deep one around $1 \mu \mathrm{m}$. Both bands are seen on the diopside sample, plus the typical pyroxene band centered at $2.3 \mu \mathrm{m}$. With increasing irradiation dose, we clearly see the darkening (14 and $9 \%$ decrease in absolute reflectance; almost $20 \%$ in relative, same order of magnitude than the $\mathrm{CV} / \mathrm{CO}$ spectra) and reddening (slope on the $0.55-2.45 \mu \mathrm{m}$ range is $0.10 \mu \mathrm{m}^{-1}$ before irradiation and goes to $0.24 \mu \mathrm{m}^{-1}$ after irradiation for the olivine, values of 0.03 and $0.15 \mu \mathrm{m}^{-1}$ for the diopside; calculated with a linear fit) effects on the spectra. However the band depth is slightly affected by irradiation: the $1 \mu \mathrm{m}$ band goes from 53.5 to $49 \%$ depth on the olivine, and from 23 to $20.5 \%$ on the diopside.

If we compare our results to other studies, olivine samples have been irradiated with $\mathrm{He}^{+}$by Fu et al. [2012] and $\mathrm{Ar}^{+}$by Brunetto et al. [2006]. The first authors used ion energy and fluence close to ours and showed a strong decrease of the reflectance in the NIR part of the spectrum, while here we only notice the decrease within the VIS range. The $1 \mu \mathrm{m}$ band also suffered a stronger decrease in depth (from 30 to $15 \%$ ). The second authors used higher energy (200 keV) but lower fluence (factor 10) and the reflectance decrease mainly happens in the VIS. They also performedion irradiation of an orthopyroxene and our results are consistent (one has to notice that here we study a clinopyroxene: bands are not located at the same wavelengths). Furthermore, Kohout et al. [2014] published a VIS-NIR spectroscopic study of several olivine samples containing different amount of $n p F e^{0}$ (artificially produced) and showed that these particles induce a slope reddening, a reflectance decrease and the mitigation of the band. We plan to probe our samples using TEM to highlight their possible presence after irradiation. 
The silicate MIR spectra (see Fig. A2) before irradiation are in agreement with literature data [Salisbury, 1991]. On the olivine, four bands are detected (attributed to $\mathrm{SiO}_{4}$ symmetric and antisymmetric stretchings [Jaeger et al., 1998]) and locations correspond to a Forsterite number of Fo $\approx 70-80$ [Hamilton, 2010]. After irradiation, we notice a shift toward longer wavelengths and Fo $\approx 50-60$ (iron-rich region). The pyroxene signatures on the diopside spectra move toward the same region. The band displacement is not the only effect observed here: the most intense peak on olivine suffers a distorsion while some peaks on diopside vanish after irradiation. The mineral structure is strongly affected. As cited in the main text, and as proposed by Brunetto et al.[2014], this shift toward longer wavelengths could be due to a preferential Mg loss within minerals. Demyk et al. [2001] and Carrez et al. [2002] noticed amorphization of olivine $(F o=90)$ and decrease of $\mathrm{Mg} / \mathrm{Si}$ ratio after $\mathrm{He}^{+}$irradiation (at the same energy than used in this study), and Demyk et al. [2004] saw the same amorphization effect on pyroxenes. One has to note that Carrez et al. [2002] proposed that a reduction process leading to formation of $\mathrm{nFe}^{0}$ could also explain the surface enrichment in $\mathrm{Fe}$.

\section{Appendix B. Pellet vs. powder spectra}

The Fig. B1 shows bidirectional reflectance spectra $\left(i=45^{\circ}\right.$ and $\left.\mathrm{e}=0^{\circ}\right)$ of the Allende meteorite (provided by L. Folco, Museo Nazionale dell'Antartide, Siena, Italy; same sample as the one presented in Brunetto et al. [2014]). We took spectra on the powder (grain size 1-100 $\mu \mathrm{m}$ ) and on a pellet made from that powder. Several differences can be noticed. First, the pellet spectrum is brighter (reflectance of $13 \%$ at $0.55 \mu \mathrm{m})$ than the powder one $(7.5 \%)$. This is because the pressed pellet has a smoother surface and acts almost like a mirror. Second, the slope is redder 
on the powder (the cause of a blue slope in solid samples is also due to surface roughness). The spectral variations observed with respect to sample preparation effects are addressed in Cloutis et al. [2012b] and references therein. Another example of variation between pellet and powder can be found by comparing our Tagish Lake VISNIR spectrum before irradiation and those measured on powder by Izawa et al. [2015]. These authors find redder slopes than ours but reflectance values are comparable.

\section{References}

Beck P., Quirico E., Montes-Hernandez G., et al., Aug. 2010, Geochimica et Cosmochimica Acta, 74, 4881

Beck P., Pommerol A., Thomas N., et al., Mar. 2012, Icarus, 218, 364

Beck P., Garenne A., Quirico E., et al., Feb. 2014, Icarus, 229, 263

Berger E.L., Keller L.P., Mar. 2015, In: Lunar and Planetary Science Conference, vol. 46 of Lunar and Planetary Inst. Technical Report, 2351

Blinova A.I.,Herd C.D.K., Duke M.J.M., 2014a, Meteoritics \& Planetary Science, 49,1100,URL http: //dx . doi . org/10 . 1111/maps . 12303

Blinova A.I., Zega T.J., Herd C.D.K., Stroud R.M., 2014b, Meteoritics \& Planetary Science, 49, 473, URL http: //dx . doi .org/10 . 1111/maps . 12271

Bonal L., Brunetto R., Beck P., et al., Sep. 2015, Meteoritics and Planetary Science, 50,1562 
Brearley A.J., 2006, The Action of Water, 584-624

Brucato J.R., Strazzulla G., Baratta G., Colangeli L., Jan. 2004, Astronomy and Astrophysics, 413, 395

Brunetto R., Roush T.L., Apr. 2008, Astronomy \& Astrophysics, 481, 879

Brunetto R., Strazzulla G., Dec. 2005, Icarus, 179, 265

Brunetto R., Vernazza P., Marchi S., et al., Oct. 2006, Icarus, 184, 327

Brunetto R., Roush T.L., Marra A.C., Orofino V., Nov. 2007, Icarus, 191, 381

Brunetto R., Borg J., Dartois E., et al., Apr. 2011, Icarus, 212, 896

Brunetto R., Lantz C., Ledu D., et al., 2014, Icarus, 237, 278

Brunetto R., Loeffler M.J., Nesvorny D., Sasaki S., Strazzulla G., 2015, In: Michel P., DeMeo F.E., Bottke Jr. (eds.) Asteroids IV, 597-616

Bus S.J., Binzel R.P., Ju1. 2002, Icarus, 158, 146

Carrez P., Demyk K., Cordier P., et al., Nov. 2002, Meteoritics and Planetary Science, 37,1599

Clark B.E., Veyerka J., Helfenstein P., et al., Jul. 1999, Icarus, 140, 53

Clark B.E., Lucey P., Helfenstein P., et al., Dec. 2001, Meteoritics and Planetary Science, 36, 1617

Clark B.E., Hapke B., Pieters C., Britt D., 2002a, In: Bottke Jr. W.F., Cellino A., Paolicchi P., Binzel R.P. (eds.) Asteroids III, 585-599 
Clark B.E., Helfenstein P., Bell J.F., et al., Jan. 2002b, Icarus, 155, 189

Clark R.N., Swayze G.A., Wise R., et al., Dec. 2007, U.S. Geological Survey, Digital Data Series, 231

Cloutis E.A., Hiroi T., Gaffey M.J., Alexander C.M.O., Mann P., Mar. 2011a, Icarus, 212, 180

Cloutis E.A., Hudon P., Hiroi T., Gaffey M.J., Mann P., Nov. 2011b, Icarus, 216, 309

Cloutis E.A., Hudon P., Hiroi T., Gaffey M.J., Mann P., Aug. 2012a, Icarus, 220, 466

Cloutis E.A., Hudon P., Hiroi T., et al., Sep. 2012b, Icarus, 221, 328

de Sanctis M.C., Coradini A., Ammannito E., et al., Dec. 2011, Space Science Reviews, 163, 329

Delbo M., Libourel G.,Wilkerson J., et al., Apr. 2014, Nature, 508, 233

DeMeo F.E., Binzel R.P., Slivan S.M., Bus S.J., Jul. 2009, Icarus, 202, 160

Demyk K., Carrez P., Leroux H., et al., Mar. 2001, Astronomy and Astrophysics, $368, \mathbf{L} 38$

Demyk K., d'Hendecourt L., Leroux H., Jones A.P., Borg J., Jun. 2004, Astronomy and Astrophysics, 420, 233

Dukes C.A., Fulvio D., Baragiola R.A., Nov. 2015, In: Space Weathering of Airless Bodies: An Integration of Remote Sensing Data, Laboratory Experiments and Sample Analysis Workshop, vol. 1878 of LPI Contributions, 2063 
Dumas P., Polack F., Lagarde B., et al., 2006, Infrared Physics \& Technology, 49, 152

Emery J.P., Cruikshank D.P., van Cleve J., Stansberry J.A., Mar. 2005, In: 36th Annual Lunar and Planetary Science Conference

Ferrari A.C., Robertson J., Aug. 2001, Physical Review B, 64, 075414

Fornasier S., Hasselmann P.H., Barucci M.A., et al., Nov. 2015, Astonomy \& Astrophysics, 583, A30

Fornasier S., Lantz C., Perna D., et al., May 2016, Tcarus, 269, 1

Fu X., Zou Y., Zheng Y., Ouyang Z., Jun. 2012, Icarus, 219, 630

Gillis-Davis J.J., Gasda P.J., Bradley J.P., Ishii H.A., Bussey D.B.J., 2015, In: Lunar and Planetary Science Conference

Godard M., Féraud G., Chabot M., et al., 2011, Astronomy and Astrophysics, 529, A146

Gounelle M., Zolensky M.E., Tonui E., Mikouchi T., Mar. 2001, In: Lunar and Planetary Science Conference, vol. 32, 1616

Gradie J., Veverka J., Buratti B., 1980, In: Lunar and Planetary Science Conference

Grady M.M., Verchovsky A.B., Franchi I.A., Wright I.P., Pillinger C.T., May 2002, Meteoritics and Planetary Science, 37, 713

Grundy W.M., Feb. 2009, Icarus, 199, 560 
Hamilton V., Christensen P., May 2014, In: EGU General Assembly Conference Abstracts, vol. 16 of EGU General Assembly Conference Abstracts, 4687

Hamilton V.E., Mar. 2010, Chemie der Erde / Geochemistry, 70, 7

Hapke B., May 2001, Journal of Geophysical Research, 106, 10039

Hapke B., Cassidy W., Wells E., Jul. 1975, Moon, 13, 339

Hiroi T., Zolensky M.E., Pieters C.M., Sep. 2001, Science, 293, 2234

Hiroi T., Pieters C.M., Rutherford M.J., et al., Mar. 2004, In: Mackwell S., Stansbery E. (eds.) Lunar and Planetary Science Conference, vol. 35, 1616

Hiroi T., Abe M., Kitazato K., et al., Sep. 2006, Nature, 443, 56

Hiroi T., Sasaki S., Misu T., Nakamura T., Mar. 2013, In: Lunar and Planetary Science Conference, vol. 44, 1276

Howard K.T., Benedix G.K., Bland P.A., Cressey G., Aug. 2009, Geochimica et Cosmochimica Acta, 73, 4576

Huss G.R., Rubin A.E., Grossman J.N., 2006, Thermal Metamorphism in Chondrites, $567-586$

Ishiguro M., Hiroi T., Tholen D.J., et al., 2007, Meteoritics and Planetary Science, 42, 1791

Izawa M.R.M., Flemming R.L., King P.L., Peterson R.C., Mar. 2008, In: Lunar and Planetary Science Conference, vol. 39, 2149 
Izawa M.R.M., FLEMMING R.L., KING P.L., PETERSON R.C., McCAUSLAND P.J.A., 2010, Meteoritics \& Planetary Science, 45, 675, URL http://dx.doi.org/10.1111/j.1945-5100.2010.01043.x

Izawa M.R.M., Craig M.A., Applin D.M., et al., Jul. 2015, Icarus, 254, 324

Jaeger C., Molster F.J., Dorschner J., et al., Nov. 1998, Astronomy and Ástrophysics, 339, 904

Jager C., Mutschke H., Henning T., Apr. 1998, Astronomy \& Astrophysics, 332, 291

Johnson P.B., Christy R.W., Jun. 1974, Physical Review B, 9, 5056

Johnson T.V., Fanale F.P., 1973, Journal of Geophysical Research, 78, 8507

Jones A.P., Apr. 2012, Astronomy and Astrophysics, 540, A2

Kaluna H.M., Gillis-Davis J.J., 2015, In: Lunar and Planetary Science Conference

Kaluna H.M., Masiero J.R., Meech K.J., Jan. 2016, Icarus, 264, 62

Keller L.P., Buseck P.R., Apr. 1990, Geochimica et Cosmochimica Acta, 54, 1155

Keller L.P., Christoffersen R., Dukes C.A., Baragiola R., Rahman Z., 2015a, In: Lunar and Planetary Science Conference

Keller L.P., Christoffersen R., Dukes C.A., Baragiola R.A., Rahman Z., Nov. 2015b, In: Space Weathering of Airless Bodies: An Integration of Remote Sensing Data, Laboratory Experiments and Sample Analysis Workshop, vol. 1878 of LPI Contributions, 2010 
Kerridge J.F., Aug. 1985, Geochimica et Cosmochimica Acta, 49, 1707

Kohout T., Čuda J., Filip J., et al., Jul. 2014, Icarus, 237, 75

Kuebler K.E., Jolliff B.L., Wang A., Haskin L.A., Dec. 2006, Geochimica et Cosmochimica Acta, 70, 6201

Lantz C., Clark B.E., Barucci M.A., Lauretta D.S., Jun. 2013, Astronomy and Astrophysics, 554, A138

Lantz C., Brunetto R., Barucci M.A., et al., May 2015, Astronomy and Astrophysics, 577, A41

Lauretta D.S., 2015, Handbook of Cosmic Hazards and Planetary Defense, chap. OSIRIS-REX Asteroid Sample-Return Mission, 543-567, Springer International Publishing, Cham, URL http://dx . doi .org/10 . 1007/978-3-319-03952-744

Lauretta D.S., Nagahara H., Alexander C.M.O., 2006, In: Lauretta D.S., McSween H.Y. (eds.) Meteorites and the Early Solar System II, 431-459

Lazzarin M., Marchi S., Moroz L.V., et al., Aug. 2006, The Astrophysical Journal Letters, 647, L179

Lim L.F., McConnochie T.H., Bell J.F., Hayward T.L., Feb. 2005, Icarus, 173, 385

Loeffler M.J., Dukes C.A., Baragiola R.A., Mar. 2009, Journal of Geophysical Research (Planets), 114, 3003

Lucey P.G., Riner M.A., Apr. 2011, Icarus, 212, 451 
Marchi S., Brunetto R., Magrin S., Lazzarin M., Gandolfi D., Dec. 2005, Astronomy and Astrophysics, 443, 769

Matsumoto T., Tsuchiyama A., Miyake A., et al., Sep. 2015, Icarus, 257, 230

Matsuoka M., Nakamura T., Kimura Y., et al., Jul. 2015, Icarus, 254, 135

Moroz L., Schade U., Wäsch R., Sep. 2000, Icarus, 147, 79

Moroz L., Baratta G., Strazzulla G., et al., Jul. 2004a, Icarus, 170, 214

Moroz L.V., Hiroi T., Shingareva T.V., et al., Mar. 2004b, In: Mackwell S., Stansbery E. (eds.) Lunar and Planetary Science Conference, vol. 35, 1279

Nagao K., Okazaki R., Nakamura T., et al., Aug. 2011, Science, 333, 1128

Nesvorný D., Jedicke R., Whiteley R.J., Ivezić Ž., Jan. 2005, Icarus, 173, 132

Noble S.K., Pieters C.M., Keller L.P., Dec. 2007, Icarus, 192, 629

Noguchi T., Nakamura T., Kimura M., et al., Aug. 2011, Science, 333, 1121

Noguchi T., Kimura M., Hashimoto T., et al., Feb. 2014, Meteoritics and Planetary Science, 49,188

Pearson V.K., Sephton M.A., Franchi I.A., Gibson J.M., Gilmour I., 2006, Meteoritics and Planetary Science, 41, 1899

Pieters C.M., Taylor L.A., Noble S.K., et al., Sep. 2000, Meteoritics and Planetary Science, 35, 1101

Pizzarello S., Cooper G.W., Flynn G.J., 2006, In: Lauretta D.S., McSween H.Y. (eds.) Meteorites and the Early Solar System II, 625-651 
Reddy V., Dunn T.L., Thomas C.A., Moskovitz N.A., Burbine T.H., 2015, In: Michel P., DeMeo F.E., Bottke Jr. (eds.) Asteroids IV

Reuter D.C., Simon-Miller A.A., Oct. 2012, In: International Workshop on Instrumentation for Planetary Missions, vol. 1683 of LPI Contributions, 1074

Richardson J.E., Melosh H.J., Greenberg R.J., O’Brien D.P., Dec. 2005, Icarus, 179,325

Rivkin A.S., Howell E.S., Vilas F., Lebofsky L.A., Mar, 2002, In: Bottke Jr. W.F., Cellino A., Paolicchi P., Binzel R.P. (eds.) Asteroids III, 235-253

Roush T.L., Mar. 2003, Meteoritics and Planetary Science, 38, 419

Rubin A.E., Wasson J.T., Jul. 1987, Geochimica et Cosmochimica Acta, 51, 1923

Salisbury J., 1991, Infrared (2.1-25 um) spectra of minerals, Johns Hopkins studies in earth and space sciences, Johns Hopkins University Press, URL http://books.google.fr/books?id=0k4SAQAAIAAJ

Salisbury J.W., D’Aria D.M., Mar. 1989, In: Lunar and Planetary Science Conference, vol. 20, 940

Sasaki S., Nákamura K., Hamabe Y., Kurahashi E., Hiroi T., Mar. 2001, Nature, 410,555

Sasaki/S., Hiroi T., Nakamura K., et al., 2002, Advances in Space Research, 29, 783

Schmitt-Kopplin P., Gabelica Z., Gougeon R.D., et al., Feb. 2010, Proceedings of the National Academy of Science, 107, 2763 
Shestopalov D.I., Golubeva L.F., Cloutis E.A., Jul. 2013, Icarus, 225, 781

Strazzulla G., Dotto E., Binzel R., et al., Mar. 2005, Icarus, 174, 31

Takagi Y., Yoshikawa M., Abe M., et al., Dec. 2011, AGU Fall Meeting Abstracts

Takir D., Emery J.P., Jun. 2012, Icarus, 219, 641

Tomeoka K., McSween H.Y. Jr., Buseck P.R., Oct. 1989, Antarctic Meteorite Research, 2, 221

Vernazza P., Fulvio D., Brunetto R., et al., Jul. 2013, Icarus, 225, 517

Walsh K.J., Richardson D.C., Michel P., Jul. 2008, Nature, 454, 188

Weisberg M.K., McCoy T.J., Krot A.N., 2006, Systematics and Evaluation of Meteorite Classification, 19-52

Yamada M., Sasaki S., Nagahara H., et al., Nov. 1999, Earth, Planets, and Space, 51,1255

Yoshikawa M., Fujiwara A., Kawaguchi J., Aug. 2007, Highlights of Astronomy, 14,323

Zolensky M., Barrett R., Browning L., Jul. 1993, Geochimica et Cosmochimica Acta, 57, 3123 

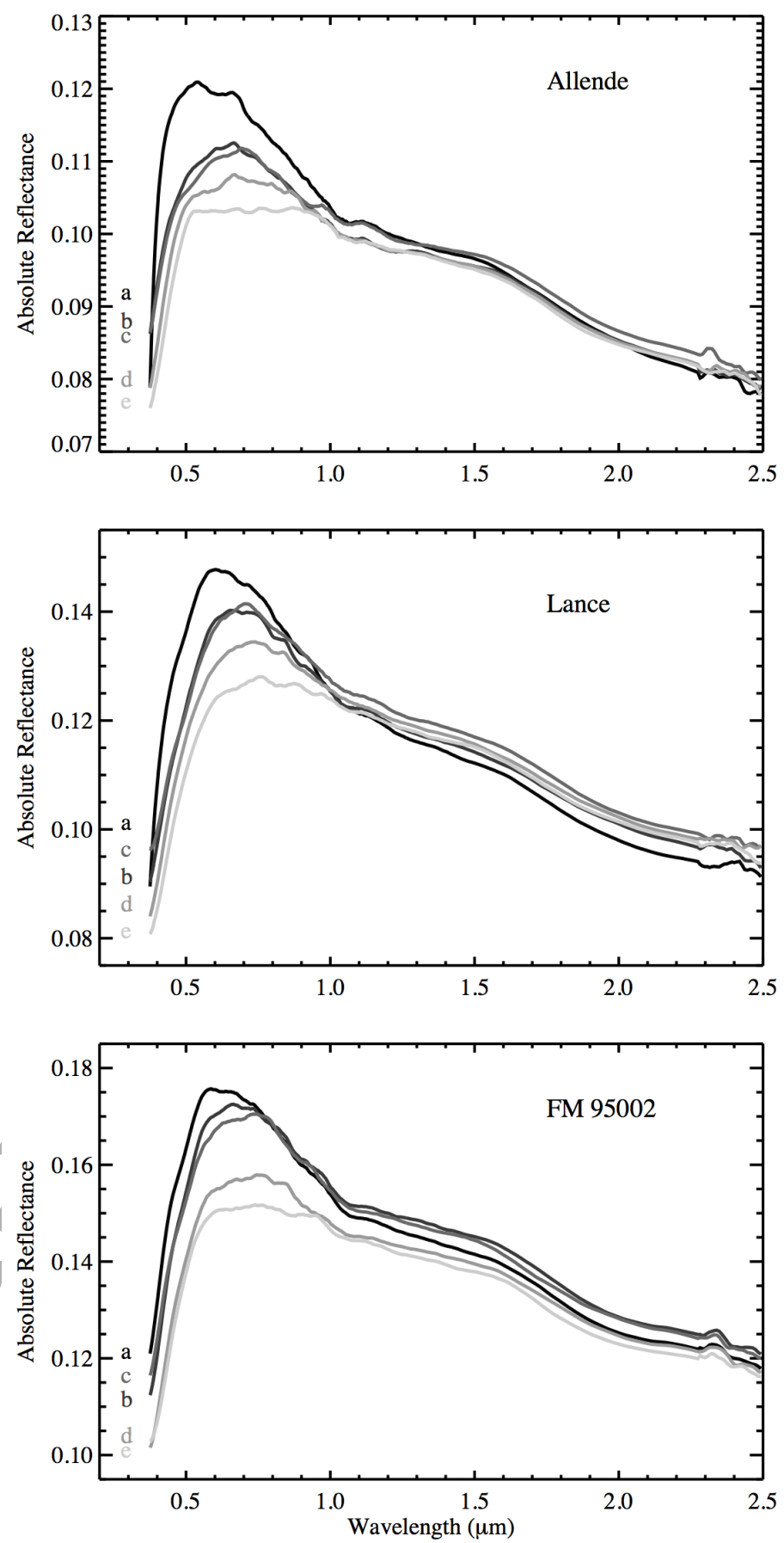

Figure 1: Visible to near-infrared bidirectional diffuse reflectance spectra of CV Allende (top), COs Lancé (middle), and Frontier Mountain $95 \$ 002$ (bottom) before (black line; a) and after irradiation with $5 \cdot 10^{15}(\mathrm{~b}), 1 \cdot 10^{16}(\mathrm{c}), 3 \cdot 10^{16}(\mathrm{~d})$, and $6 \cdot 10^{16} \mathrm{He}^{+} / \mathrm{cm}^{2}$ (lightest grey; e). 

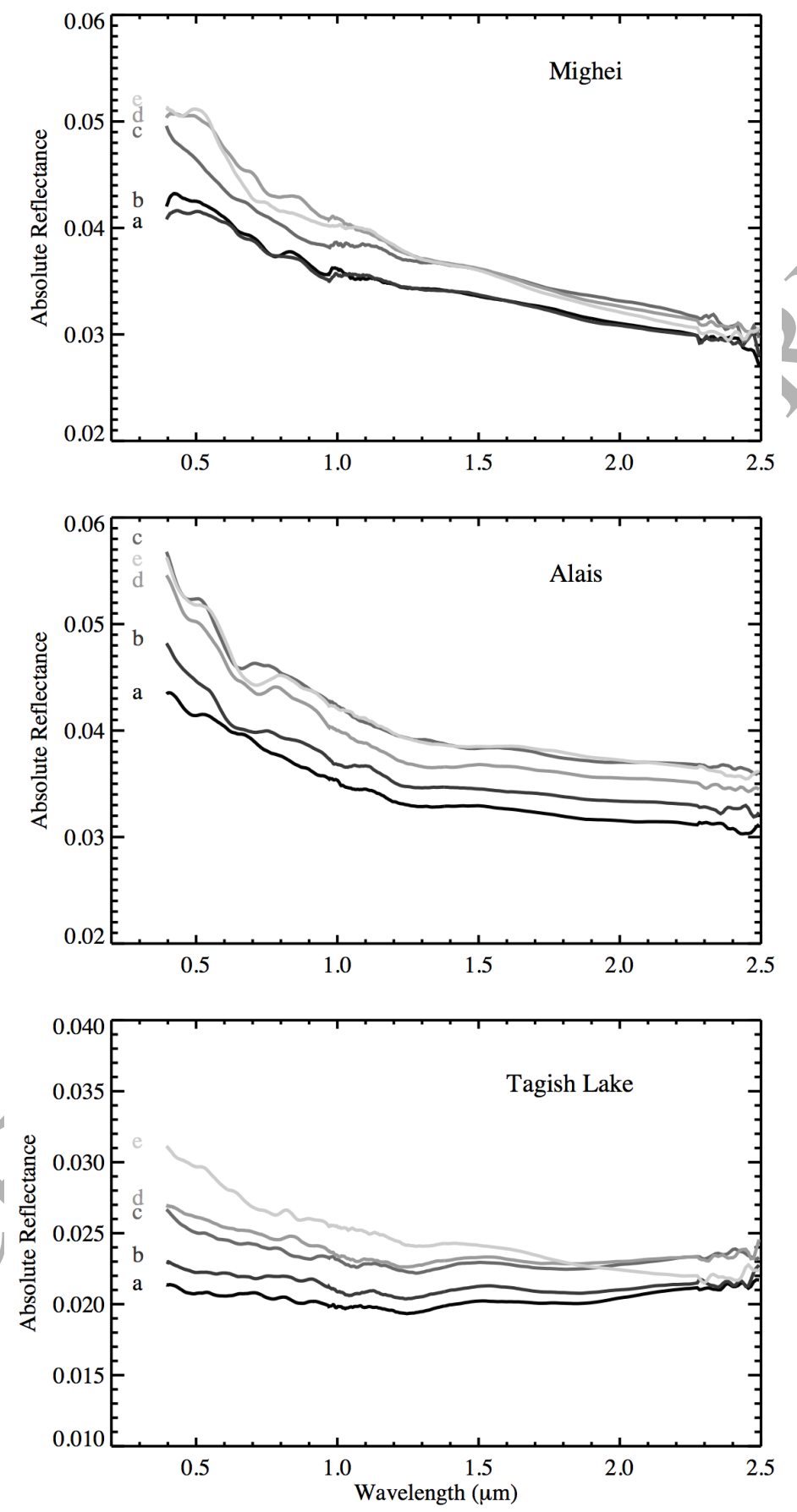

Figure 2: Same as Fig. 1 for CM Mighei (top), CI Alais (middle) and ungrouped Tagish Lake (bottom). One can note some artifacts on the spettra of Mighei and Alais (between 0.6 and $0.9 \mu \mathrm{m}$ ) probably due to the specular component of the reflected light. 

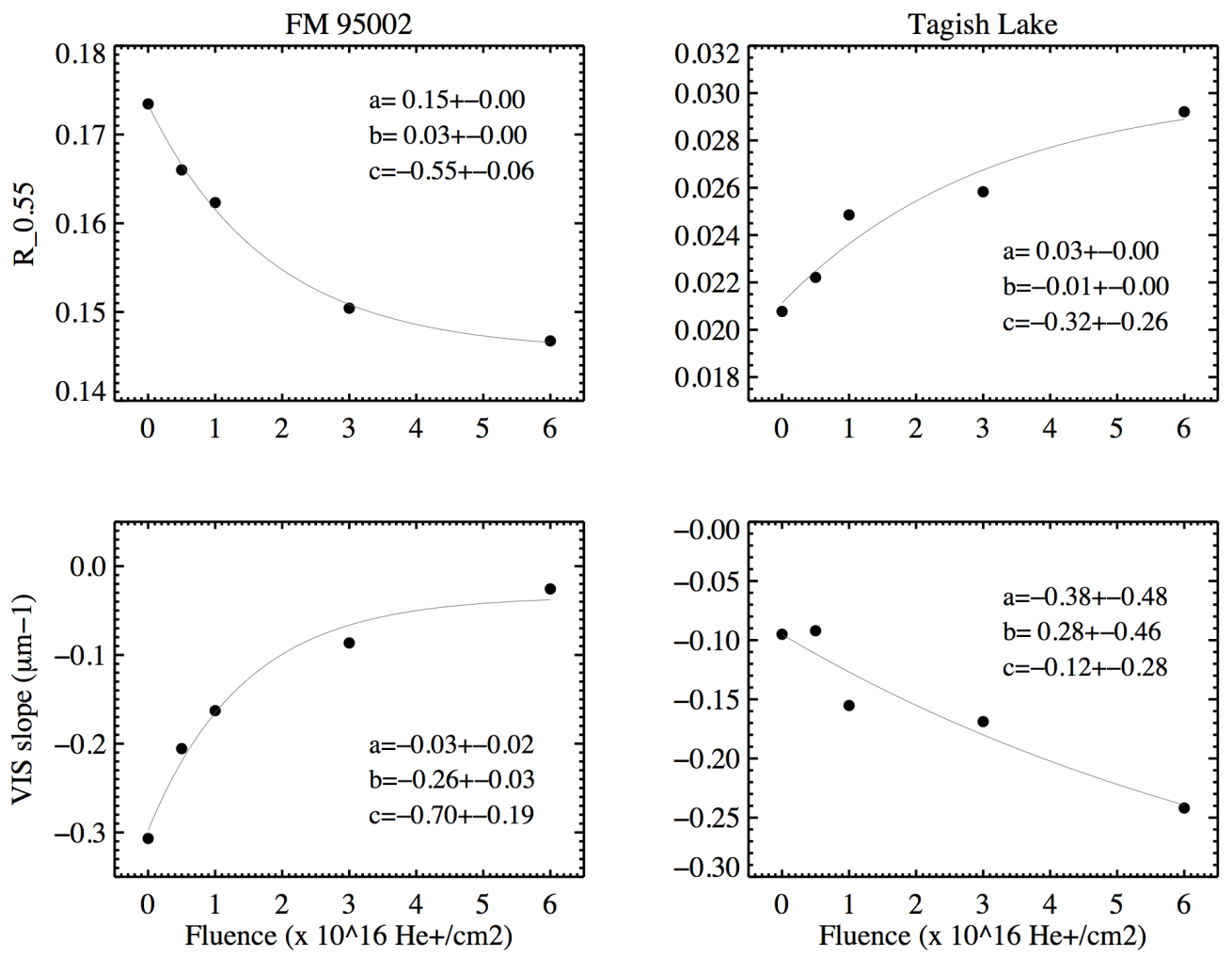

Figure 3: Absolute reflectance at $0.55 \mu \mathrm{m}$ (top) and VIS slope on the range $0.55-1.05 \mu \mathrm{m}$ (bottom) for the CO Frontien Mountain 95002 (left) and ungrouped Tagish Lake (right), the two endmembers of our set of samples, as a function of the irradiation fluence (from 0 to $6 \cdot 10^{16} \mathrm{He}^{+} / \mathrm{cm}^{2}$ ). We use an exponential fit (grey line) to describe the behavior of these CCs under ion irradiation ( $y=$ $a+b \cdot \exp (x \cdot c))$. Allende and Lancé follow the same trend as FM 95002, while Mighei and Alais behave similarly to Tagish Lake. 


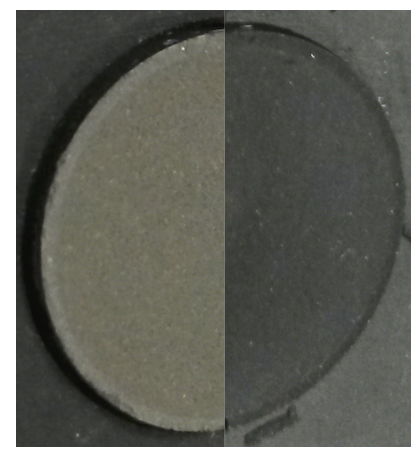

Figure 4: Pictures of Lancé (left) and Tagish Lake (right) taken after iradiation with the highest dose. One can see the thin unaltered corona at the edge of the pellets: it is brighter on Lancé so the irradiation lead to a darkening of the surface, as opposed to the Tagish Lake brightening behavior.
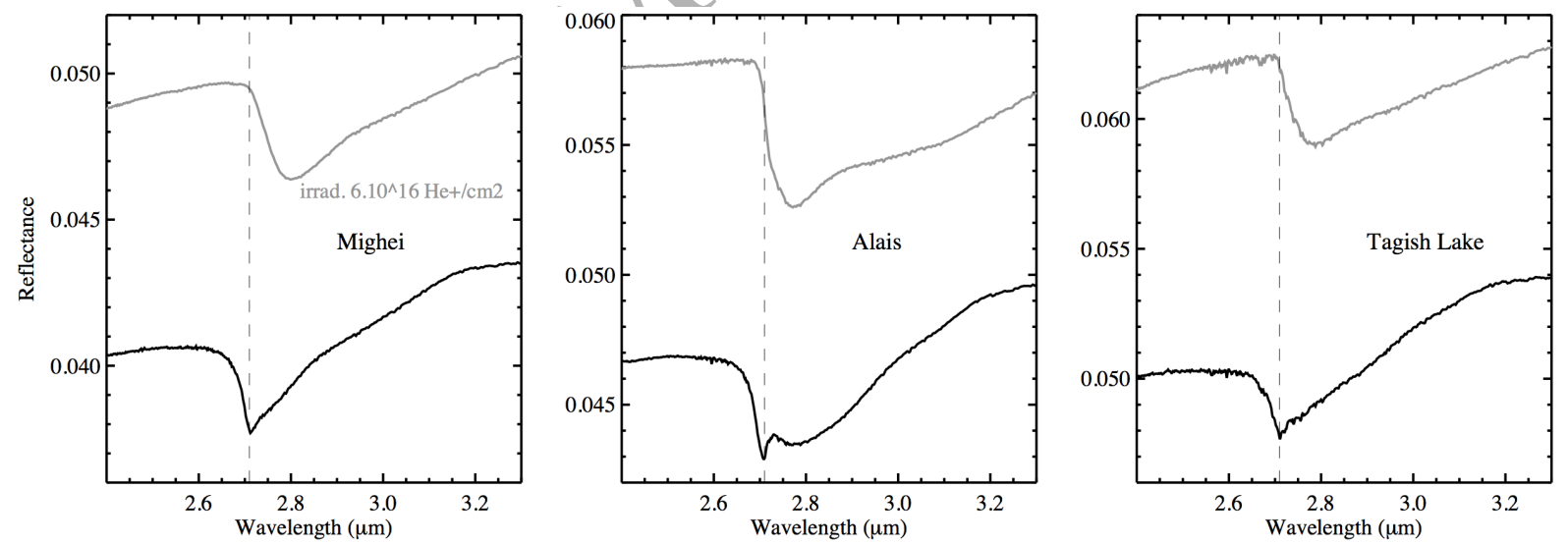

Figure 5: NIR confocal reflectance spectra around OH-stretching bands for the non-irradiated area (black line) and the highest fluence of irradiation (grey line) for Mighei (left), Alais (central), and Tagish Lake (right). The dotted line indicates the $2.71 \mu \mathrm{m}$ position on each plot corresponding to the band minimum. 

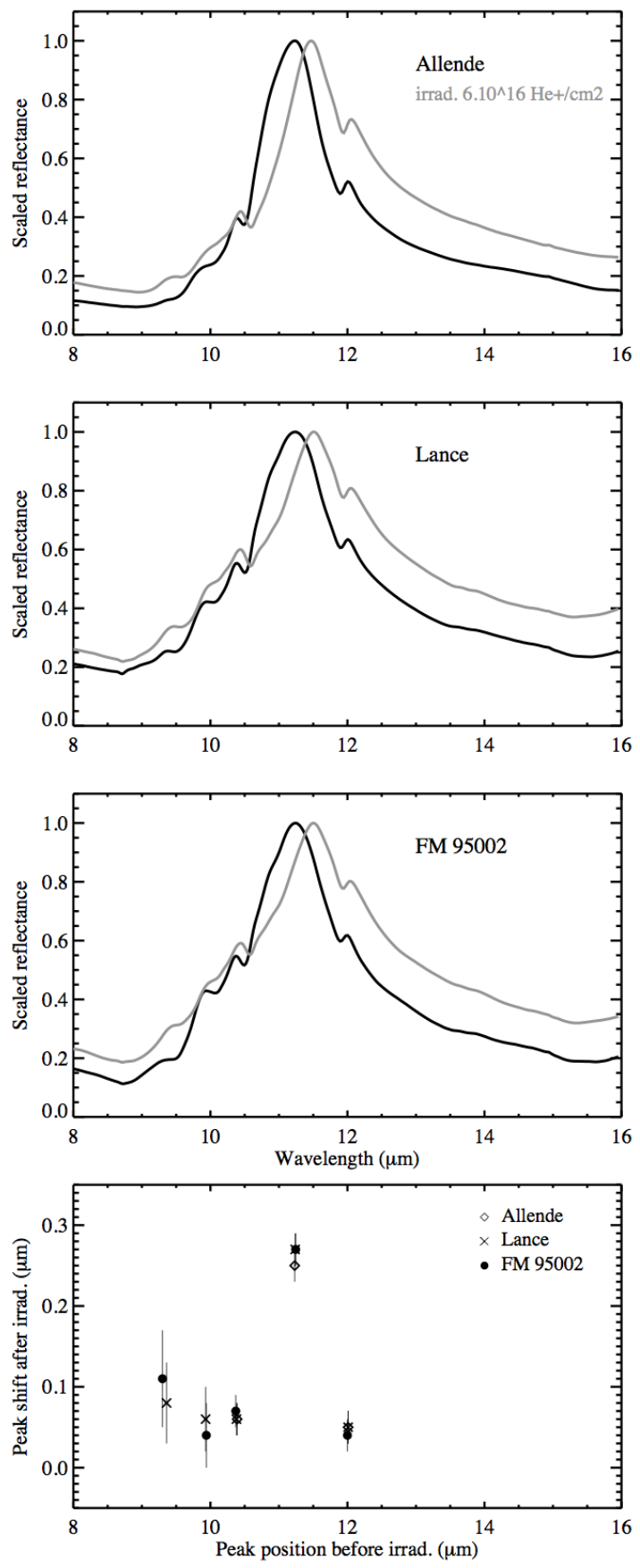

Figure 6: Mid-Infrared confocal reflectance spectra of Allende (top), Lancé (middle up), and Frontier Mountain 95002 (middle down) on a non-irradiated area of the pellets (black line), and irradiated at the highest fluence (grey line). Spectra are scaled to unity at strongest peak to facilitate the comparison. The bottom panel shows the band shift as a function of the initial position (same $\mathrm{x}$-axis than the other panels). Error on the initial position is within the symbol size. 

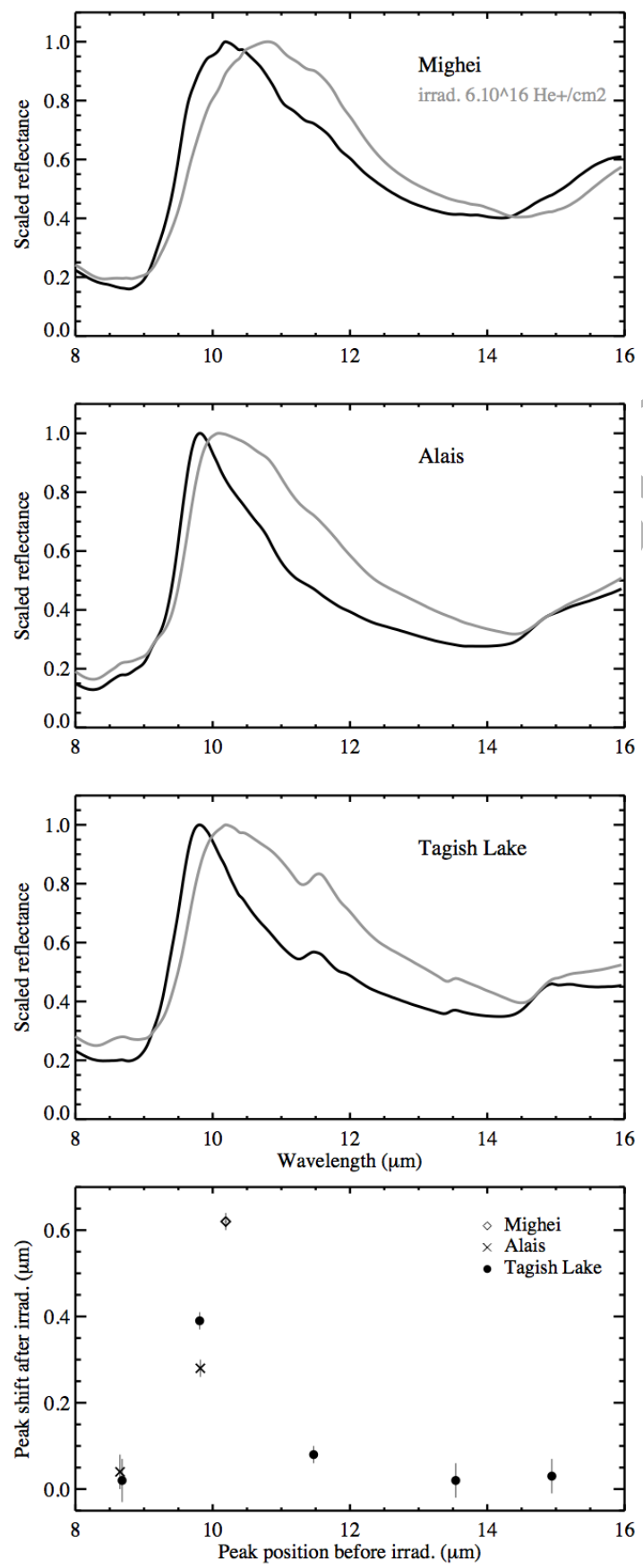

Figure 7: Same as Fig. 6 for Mighei, Alais, and Tagish Lake. 


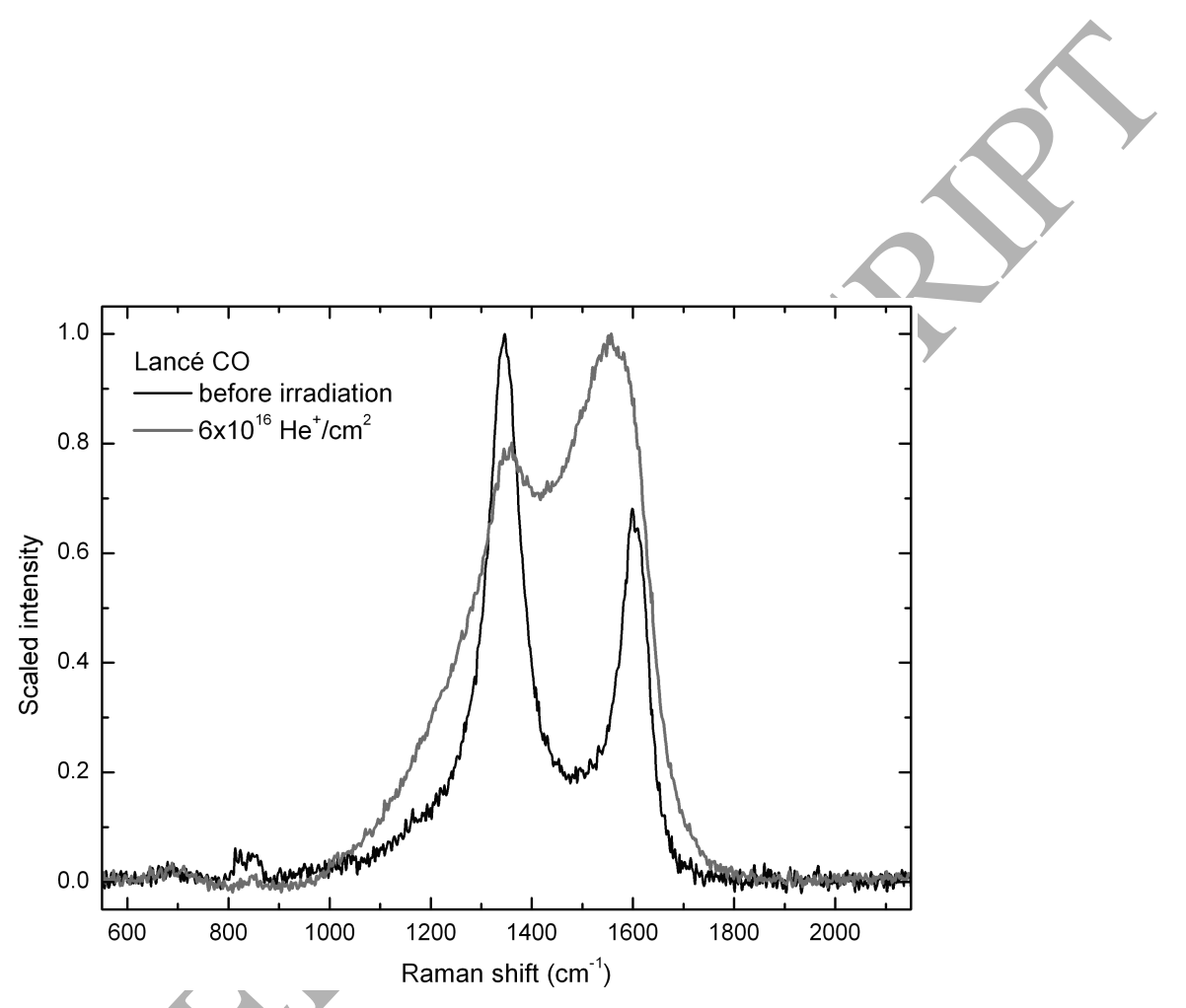

Figure 8: Raman spectra of Lancé for the unirradiated area (black line) and for irradiated area with $6 \cdot 10^{16} \mathrm{He}^{+} / \mathrm{cm}^{2}$ (grey line). The $\mathrm{D}\left(\sim 1350 \mathrm{~cm}^{-1}\right)$ and $\mathrm{G}\left(\sim 1580 \mathrm{~cm}^{-1}\right)$ bands become larger, and olivine bands $\left(\sim 820\right.$ and $\left.850 \mathrm{~cm}^{-1}\right)$ are also heavily affected by the irradiation. Spectra are scaled to unity at the strongest peak. 


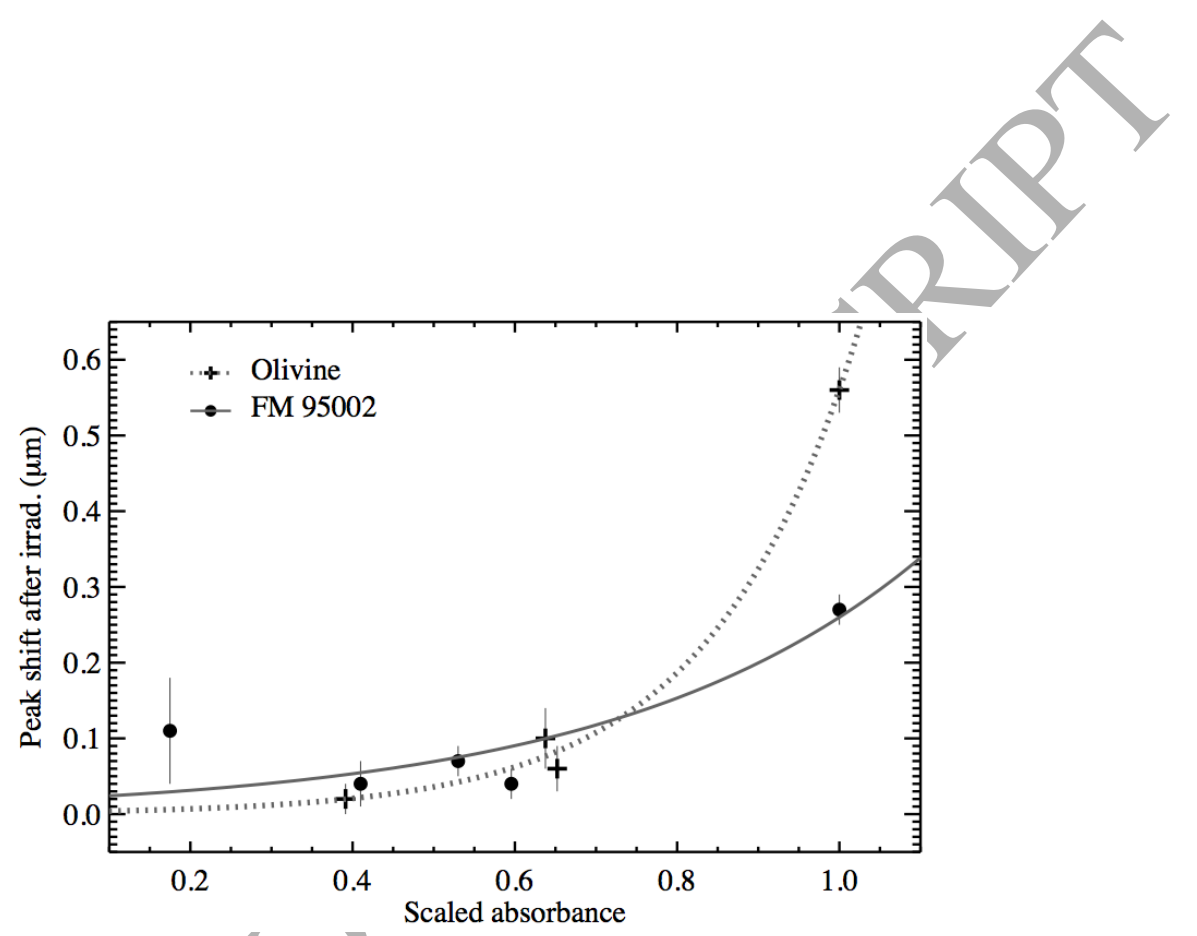

Figure 9: Band shift of features in the MIR after irradiation with the highest dose $\left(6 \cdot 10^{16} \mathrm{He}^{+} / \mathrm{cm}^{2}\right)$ for the CO Frontier Mountain 95005 and olivine samples as a function of $-\ln (1-R)$ where $R$ is the reflectance measured on the corresponding band peak before irradiation. This logarithmic quantity is proportional to the imaginary part of the refractive index, therefore to the absorbance. Data are scaled to unity at strongest peak, for both samples. Exponential curves are added to guide the eye.

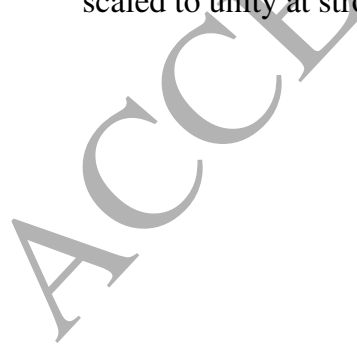



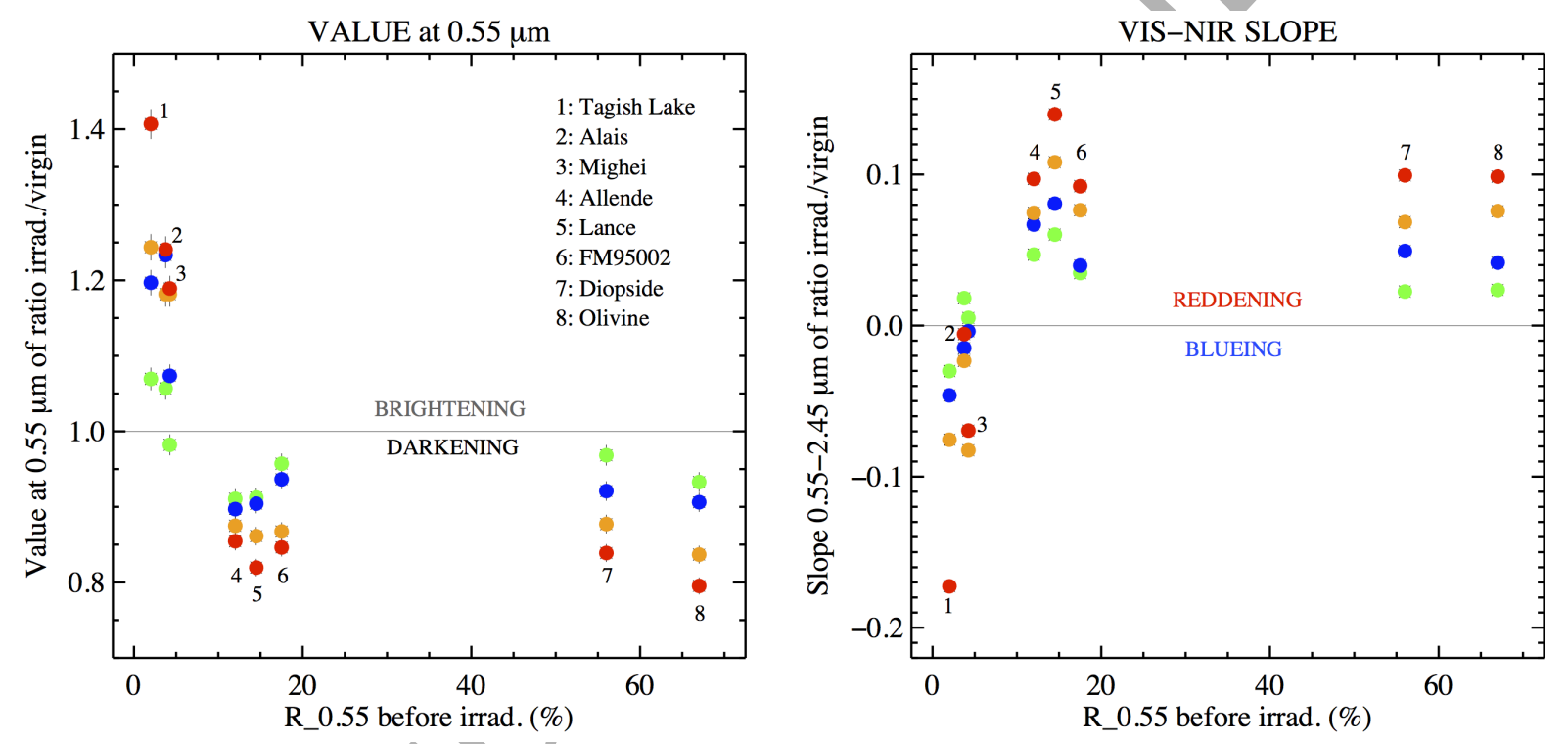

Figure 10: Value of ratioed reflectance at $0.55 \mu \mathrm{m}$ (left panel) and spectral slope calculated using a linear fit on the whole range $0.55-2.45 \mu \mathrm{m}$ (right panel) of ratios "virgin sample over irradiated sample" for each irradiation: $5 \cdot 10^{15}$ (green), $1 \cdot 10^{16}$ (blue), $3 \cdot 10^{16}$ (orange), and $6 \cdot 10^{16} \mathrm{He}^{+} / \mathrm{cm}^{2}$ (red). The error bar on $\mathrm{R}_{0.55}$ before irradiation is within the symbol size; the same on the slope. The horizontal lines indicate the value for which the ratio reflectance value and slope do not change after irradiation. 


\begin{tabular}{|c|c|c|c|}
\hline Sample & Experiment & Effect & Reference \\
\hline Allende & $\begin{array}{l}\text { ion } \\
\text { ion }\end{array}$ & $\begin{array}{c}\text { red in the VIS } \\
\text { dark + red in the VIS }\end{array}$ & $\begin{array}{l}\text { Lazzarin et al. [2006] } \\
\text { Brunetto et al. [2014] }\end{array}$ \\
\hline FM 95002 & ion & red in the VIS & Lazzarin et al. [2006] \\
\hline Mighei & laser & dark + red in the VIS & Móroz et al. [2004b] \\
\hline Tagish Lake & $\begin{array}{l}\text { ion } 1 \\
\text { ion } 2 \\
\text { laser }\end{array}$ & $\begin{array}{c}\text { red in the VISNIR } \\
\text { blue in the VISNIR } \\
\text { dark + blue in the VISNIR }\end{array}$ & $\begin{array}{l}\text { Vernazza et al. [2013] } \\
\text { Hiroi et al. [2013] }\end{array}$ \\
\hline $\begin{array}{c}\text { ALH } 83108 \text { (CO3) } \\
\text { ALH } 85002(\mathrm{CK} 4) \\
\text { MAC } 88100 \text { (CM2) } \\
\text { Orgueil + Ivuna (CI1 mix.) }\end{array}$ & laser & e VISNIR & Hiroi et al. [2013] \\
\hline Murchison (CM2) & laser & $\begin{array}{c}\text { dark }+ \text { red in the NIR } \\
\text { small changes } \\
\text { dark + blue in the VISNIR }\end{array}$ & $\begin{array}{c}\text { Keller et al. [2015a] } \\
\text { Lantz et al. [2015] } \\
\text { Matsuoka et al. [2015] }\end{array}$ \\
\hline
\end{tabular}

Table 1: Resume of SpWe laboratory experiments on slopes and reflectance. These are made on pellets, except Keller et al. [2015a] who worked on a thin section. Red and Blue refer to reddening and blueing of the spectral slopes, while dark refers to the surface brightness decrease after irradiation. VIS for visible part of the spectrum, from 0.4 to $1.0 \mu \mathrm{m}$, NIR for the infrared to $2.5 \mu \mathrm{m}$. Vernazza et al. [2013] saw both reddening and blueing/of the slope using two different experiments. Matsuoka et al. [2015] noticed that the reflectance increases below $0.4 \mu \mathrm{m}$. In Lantz et al. [2015], the changes are smaller than either effects due to sample preparation. 


\begin{tabular}{|c|cc|cc|cc|}
\hline \multirow{2}{*}{ Sample } & \multicolumn{2}{|c|}{$\mathrm{R}_{0.55}$} & \multicolumn{2}{c|}{ VISNIR slope } & \multicolumn{2}{c|}{$\begin{array}{c}\text { VIS slope } \\
\end{array}$} \\
& before & after irrad. & before & after irrad. & before & after irrad. \\
\hline \hline FM 95002 & 0.173 & 0.147 & -0.204 & -0.128 & -0.307 & -0.026 \\
Lancé & 0.145 & 0.119 & -0.245 & -0.137 & -0.364 & 0.037 \\
Allende & 0.121 & 0.103 & -0.201 & -0.124 & -0.333 & -0.021 \\
Mighei & 0.042 & 0.050 & -0.155 & -0.191 & -0.322 & -0.361 \\
Alais & 0.041 & 0.051 & -0.152 & -0.149 & -0.328 & -0.275 \\
Tagish Lake & 0.021 & 0.029 & -0.009 & -0.130 & -0.095 & -0.242 \\
\hline
\end{tabular}

Table 2: Evolution of VIS-NIR spectral parameters before and after irradiation with the highest dose $\left(6 \cdot 10^{16} \mathrm{He}^{+} / \mathrm{cm}^{2}\right) . \mathrm{R}_{0.55}$ is the absolute reflectance value at $0.55 \mu \mathrm{m}$. The slopes $\left(\mu \mathrm{m}^{-1}\right)$ are evaluated with a linear fit on the whole range $0.55-2.45 \mu \mathrm{m}$ (VISNIR), and on the range $0.55-1.05 \mu \mathrm{m}$ (VIS). The associated error on $\mathrm{R}_{0.55}$ is about $1 \%$ of the given absolute value, and less than $0.01 \mu \mathrm{m}^{-1}$ for the slopes. 


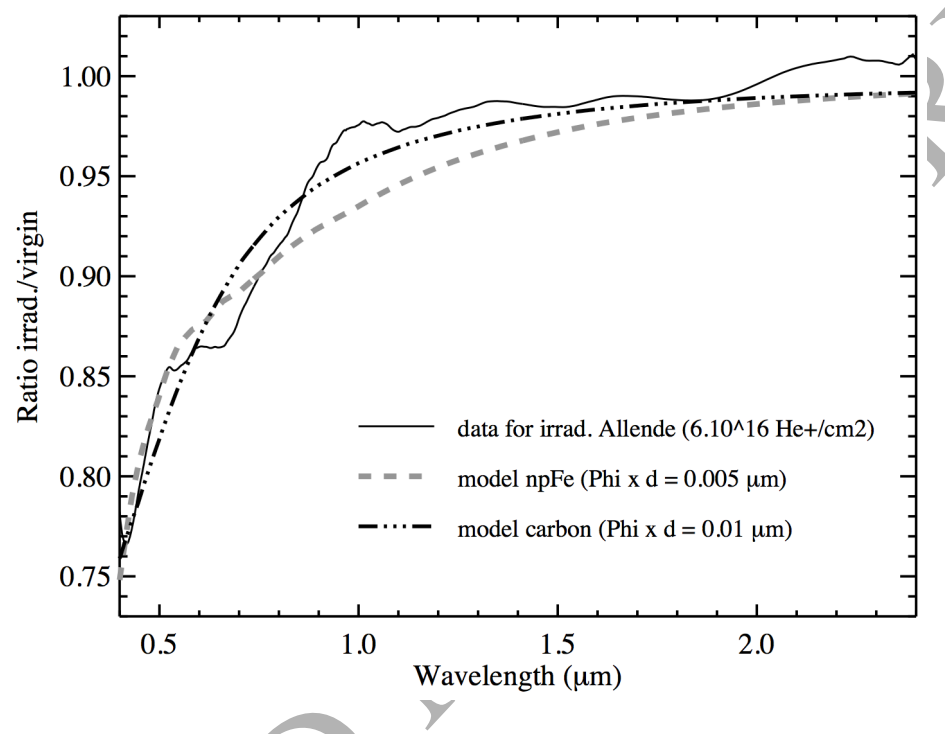

Figure 11: VIS-NIR spectrum of the meteorite Allende obtained after the irradiation with the highest fluence $\left(6 \cdot 10^{16} \mathrm{He}^{+} / \mathrm{cm}^{2}\right)$ divided by the spectrum before irradiation, called ratio (black line). The ratio is also made with a derived irradiated spectrum taking into account the Hapke model: with iron (dashed grey line) or carbon (dashed/dotted black line) inclusions. The computation gives a value $\phi \cdot d$, where $\phi$ is the volume fraction of inclusions in the host matrix and is a function of the concentration of inclusions and relative densities of inclusion and host materials, and $d$ is the mean optical path length. Here $d$ is estimated to be the same for both kinds of inclusions, so that more carbon is necessary to fit the SpWe effects. 


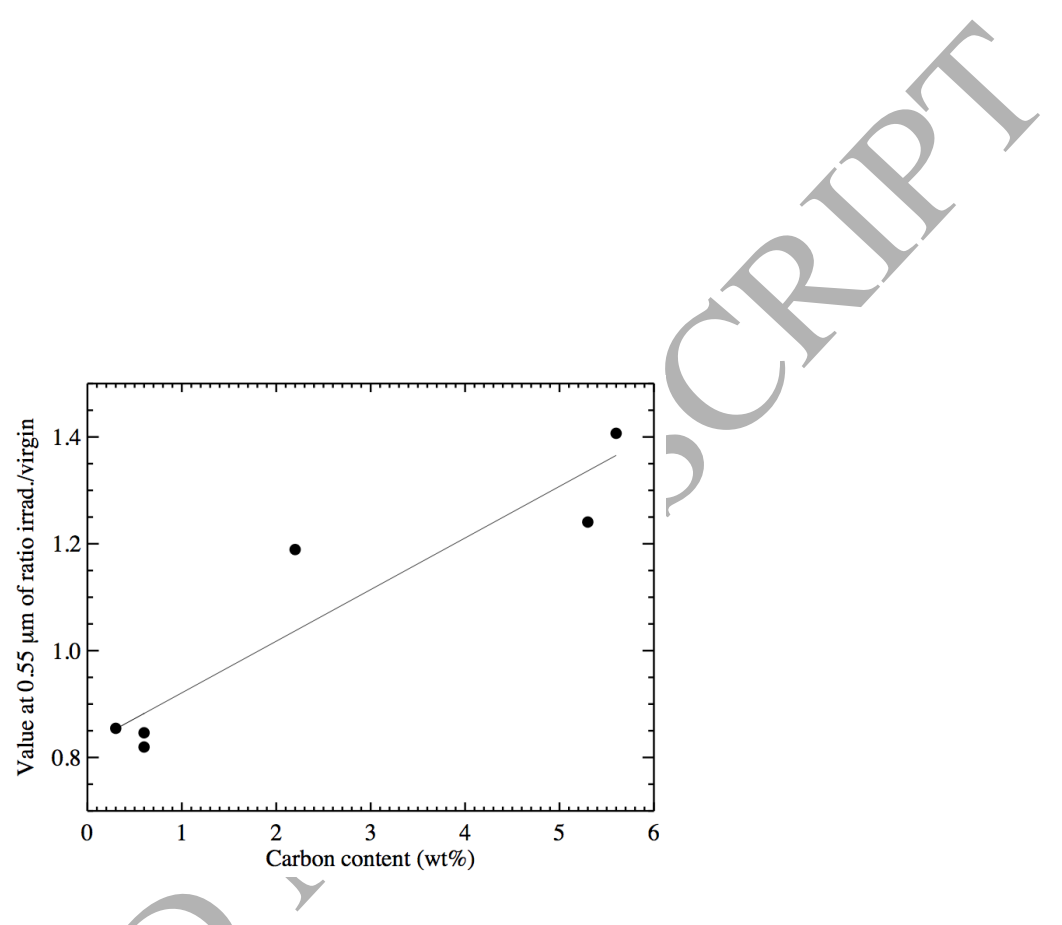

Figure 12: Value of ratioed reflectance at $0.55 \mu \mathrm{m}$ of ratios "irradiated sample over virgin sample" for the highest dose of irradiation $\left(6 \cdot 10^{16} \mathrm{He}^{+} / \mathrm{cm}^{2}\right)$ as a function of the carbon content in $\mathrm{CC}$ samples. The error bars on the carbon content are certainly high considering that we extracted values from literature: our study is made on a small piece of each $\mathrm{CC}$ but might be not representative of the whole meteorite. 

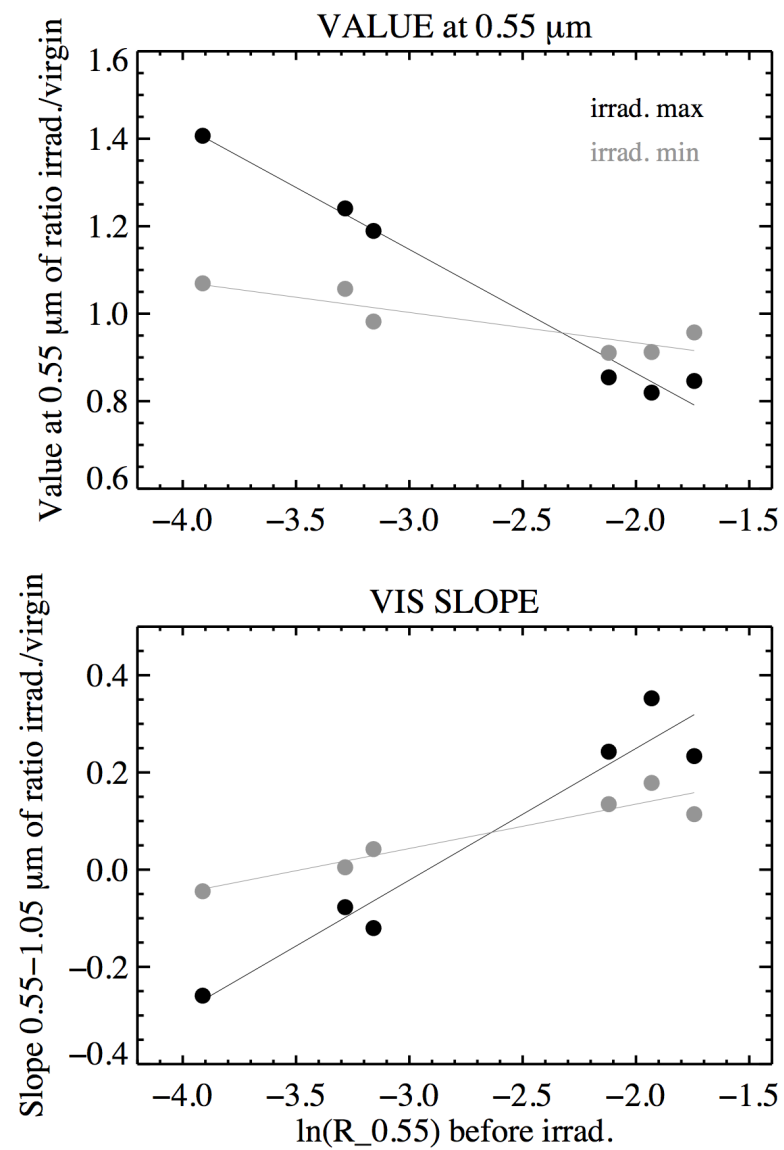

Figure 13: A linear fit $(y=a+b \cdot \ln (x))$ is used to describe the space weathering effects on carbonaceous chondrites as a function of the natural logarithm of the initial value of reflectance at $0.55 \mu \mathrm{m}$ before irradiation $\left(\mathrm{R}_{0.55}\right)$ : values of reflectance at $0.55 \mu \mathrm{m}$ (top panel) and spectral slope on the range $0.55-1.05 \mu \mathrm{m}$ (bottom panel) extracted from ratio plots. The reflectance increases for the darkest CCs, and decreases for the brightest ones, while slope gets bluer and redder respectively. This trends are accentuated with increasing dose of irradiation: here we show the first $\left(5 \cdot 10^{15} \mathrm{He}^{+} / \mathrm{cm}^{2}\right.$; grey data) and last $\left(6 \cdot 10^{16} \mathrm{He}^{+} / \mathrm{cm}^{2}\right.$; dark data) steps. See Table 3 for the equation parameters. See Fig. 14 for the behavior of parameter $b$ (related to fit slope) as a function of the irradiation dose. These fits allow us to derive the values of initial $\mathrm{R}_{0.55}$ for which we could expect no effect of space weathering on primitive bodies: by resolving the equation $y=1$ for the reflectance or $y=0$ for the slope, we find 8.2 and $5.3 \%$ respectively (fit with the highest dose). Errors estimation brings us to consider the whole range $4.5-9 \%$ 


\begin{tabular}{|cc|cc|}
\hline Parameter & \multicolumn{2}{|c|}{ Fluence $\left(\mathrm{He}^{+} / \mathrm{cm}^{2}\right)$} \\
& & min. $\left(5 \cdot 10^{15}\right)$ & max. $\left(6 \cdot 10^{16}\right)$ \\
\hline \hline \multirow{2}{*}{ Reflectance } & $\mathrm{a}$ & $0.79 \pm 0.05$ & $0.30 \pm 0.05$ \\
& $\mathrm{~b}$ & $-0.07 \pm 0.02$ & $-0.28 \pm 0.02$ \\
\hline \multirow{2}{*}{ VIS slope } & $\mathrm{a}$ & $0.32 \pm 0.04$ & $0.79 \pm 0.1$ \\
& $\mathrm{~b}$ & $0.09 \pm 0.02$ & $0.27 \pm 0.03$ \\
\hline \hline \multirow{2}{*}{ VISNIR slope } & $\mathrm{a}$ & $0.12 \pm 0.02$ & $0.38 \pm 0.01$ \\
& $\mathrm{~b}$ & $0.04 \pm 0.01$ & $0.14 \pm 0.02$ \\
\hline \multirow{2}{*}{ NIR slope } & $\mathrm{a}$ & $0.06 \pm 0.02$ & $0.20 \pm 0.07$ \\
\hline
\end{tabular}

Table 3: Values of the parameters for the linear fit $\mathrm{Val}=a+b \cdot \ln \left(R_{0.55}\right)$ with the minimal and maximal irradiation doses. We give the equation for all the tests we made: reflectance, and VIS, VISNIR and NIR slopes. Among the slopes, we chose to visually present only the VIS one in Fig. 13 because this is the most affected spectral parameter. 


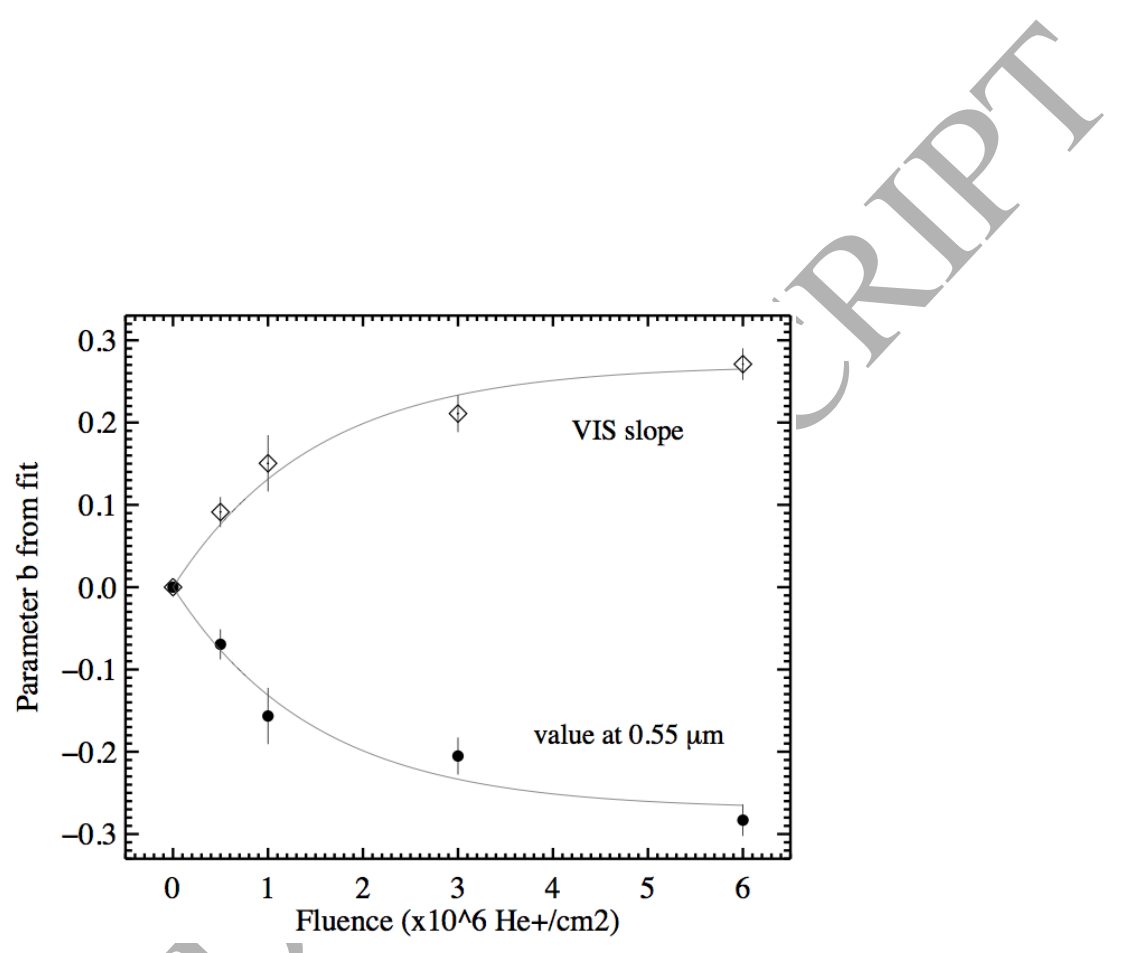

Figure 14: Evolution of the parameter $b$, related to the slope of the fit from Fig. 13, with the irradiation fluence (from 0 to $6 \cdot 10^{16} \mathrm{He}^{+} / \mathrm{cm}^{2}$ ). We show data for the ratio value at $0.55 \mu \mathrm{m}$ and the ratio slope on the range $0.55-1.05 \mu \mathrm{m}$. Both quantities seem to follow the same trend: this is because we are evaluating the reflectance and the slope from the point at $0.55 \mu \mathrm{m}$, and there is almost no change in the NIR, so we obtain the same evolution. 

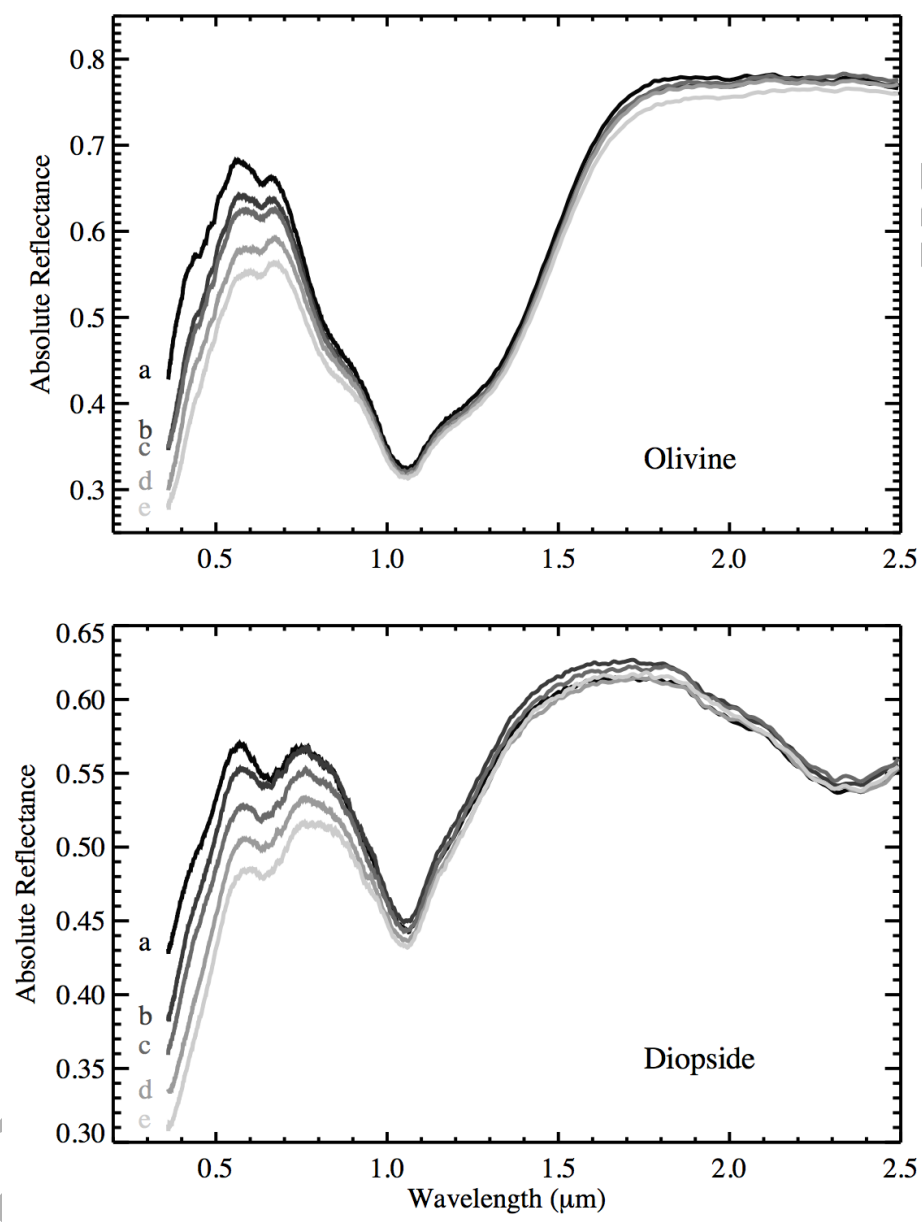

Figure 15: FIG A1 Visible to near-infrared bidirectional diffuse reflectance spectra of silicates: olivine (top) and diopside (bottom) before (black line; a) and after irradiation with $5 \cdot 10^{15}$ (b), $1 \cdot 10^{16}(\mathrm{c}), 3 \cdot 10^{16}(\mathrm{~d})$, and $6 \cdot 10^{16} \mathrm{He}^{+} / \mathrm{cm}^{2}$ (lightest grey; e). 

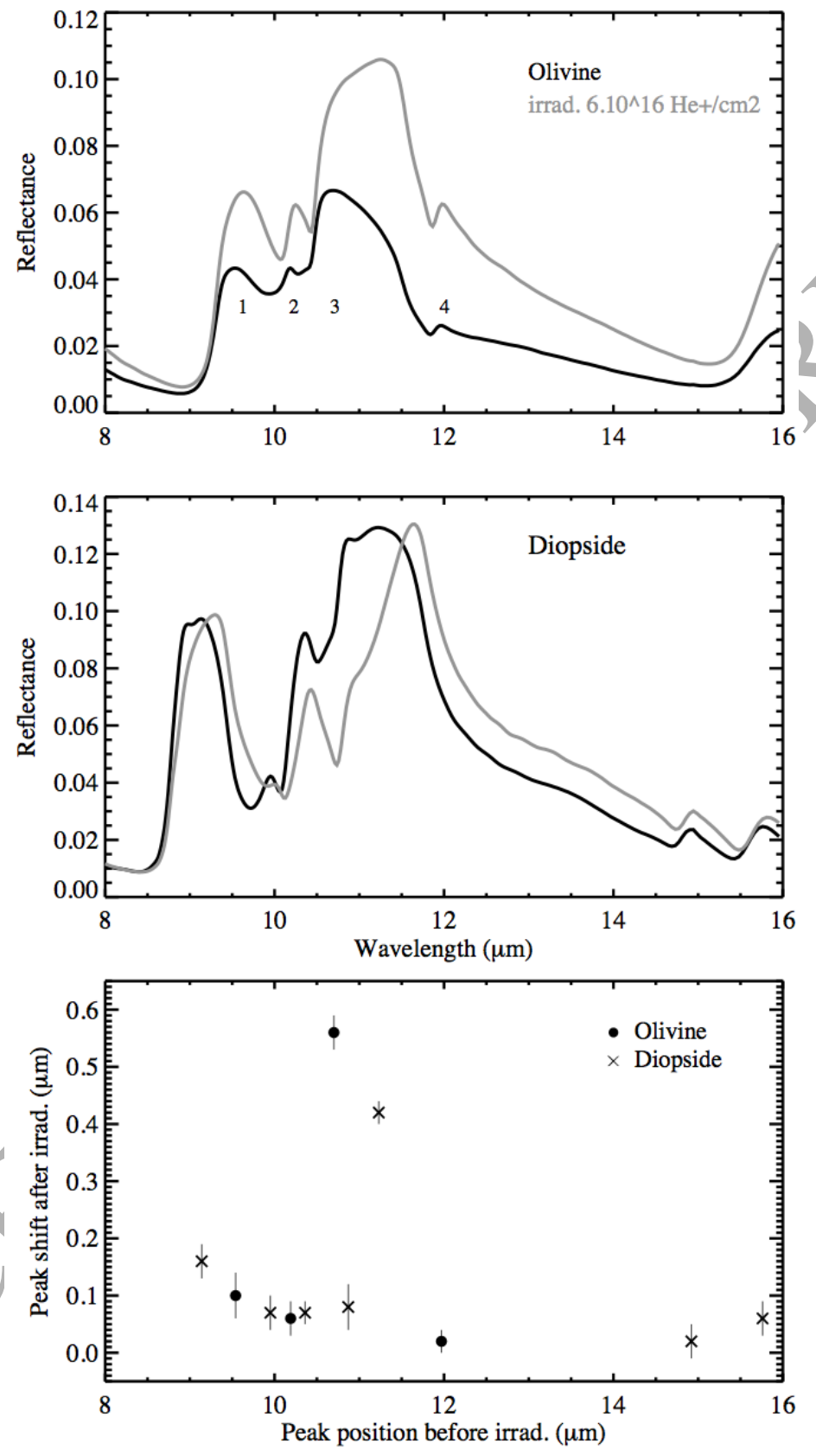

Figure 16: FIG A2 Near to Mid-Infrared confocal reflectance spectra of silicates: olivine (top) and diopside (middle) before (black line) and after irradiation with $6 \cdot 10^{16} \mathrm{He}^{+} / \mathrm{cm}^{2}$ (grey line). The bottom panel shows the band shift as a function of the initial peak position. Error on the initial position is within the symbol size. On olivine thend number as defined by [Hamilton, 2010] is given. On diopside a peak is easily seen before irradiation at $8.96 \pm 0.02 \mu \mathrm{m}$ that decreases after irradiation. 


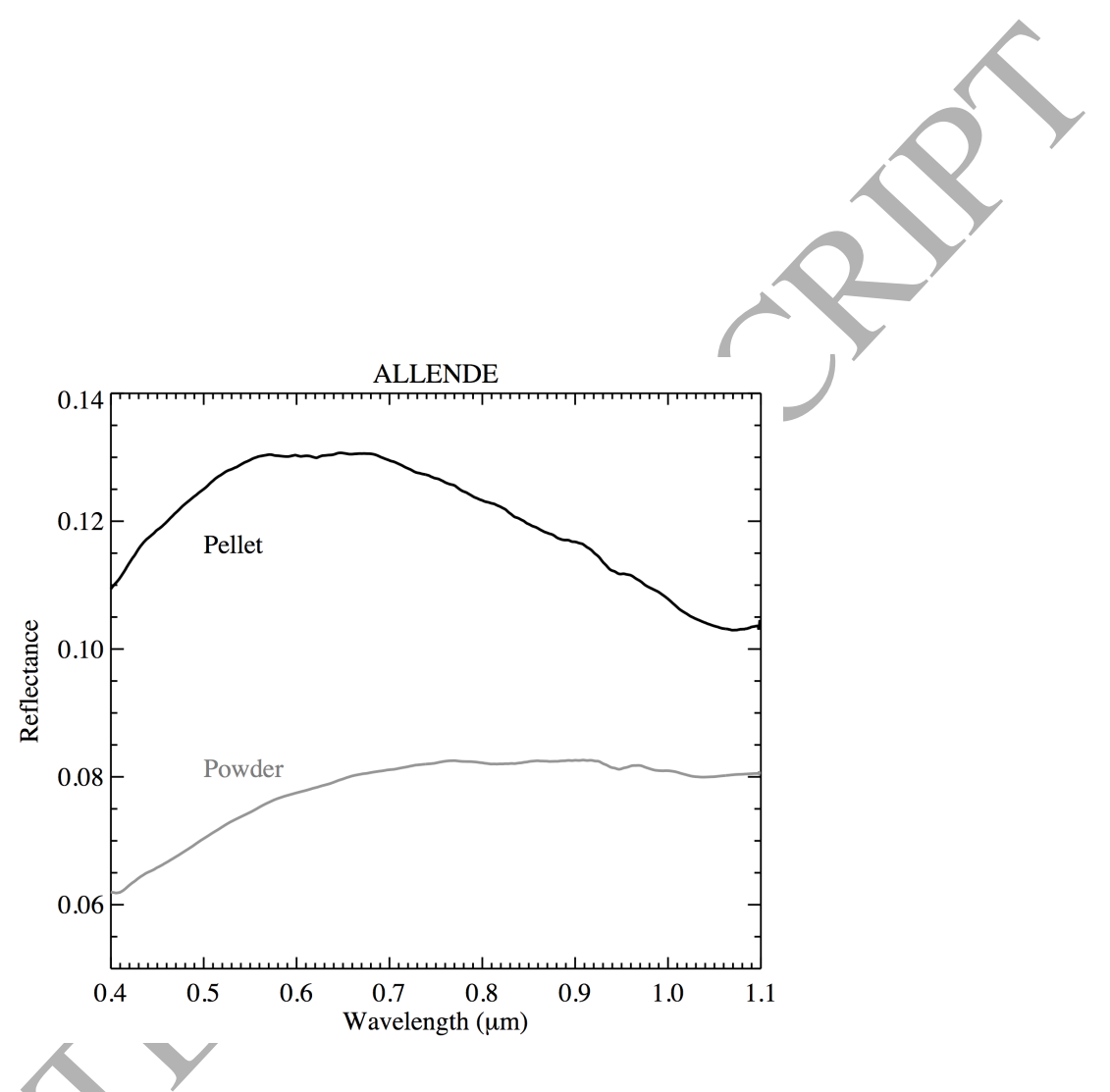

Figure 17: FIG B1 Visible bidirectional diffuse reflectance spectra of Allende: pellet (black line) and powder (grey line).

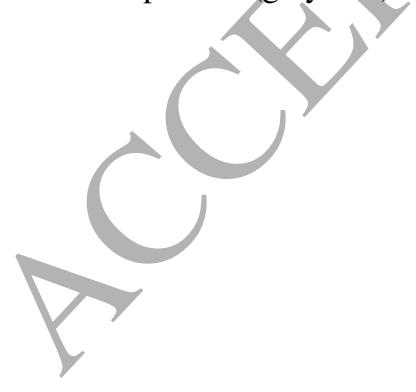

